\title{
Bird species assemblages in railway stations: variations along an urban-rural gradient
}

\author{
Anupam Chakraborty ${ }^{1}$, Sayan Das ${ }^{1}$, Anirban Ash ${ }^{2}$, Goutam K. \\ SAHA $^{1} \&$ Gautam ADITYA ${ }^{1 *}$
}

Received: June 23, 2020 - Revised: October 23, 2020 - Accepted: October 24, 2020

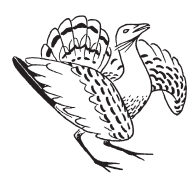

Chakraborty, A., Das, S., Ash, A., Saha, G. K. \& Aditya, G. 2020. Bird species assemblages in railway stations: variations along an urban-rural gradient. - Ornis Hungarica 28(2): 85-110. DOI: $10.2478 /$ orhu-2020-0019

\begin{abstract}
The transportation infrastructures like railway tracks and roads bear negative impacts on natural environment. However, the opposite effects are also true in some instances where the man-made constructions have positive effect on faunal assemblages. This proposition was justified through the assessment of bird species using railway stations as model man-made structures in an urban-rural gradient, in the suburbs of Kolkata, India. During the entire study period along nine different railway stations, a total of 43 bird species belonging to 12 orders and 26 families were observed. Among these, the order Passeriformes was predominant in its species composition having 18 different species from 11 different families. In urban railway stations, a total of 23 bird species under 22 genera and 14 families were observed. In suburban railway stations, a total of 35 bird species under 32 genera and 22 families were documented. The railway stations from rural region showed the maximum number of species and abundance of bird families, where a total of 36 bird species under 32 genera and 23 families were observed. The railway stations from the suburban and rural regions were more similar in species composition. Irrespective of the locations, during the entire study period, the House Crow (Corvus splendens) was the dominant species followed by the Common Myna (Acridotheres tristis). About 18 bird species exhibited a decreasing population trend observed through the global population trend analysis. In all the railway stations, the abundance of omnivores were dominant while, the number of granivores were higher in the rural regions and the nectarivores were absent in the urban regions. It was apparent that the railway stations bear a positive effect on the bird species assemblages, which can be sustained through proper environmental management planning inclusive of urban greening.
\end{abstract}

Keywords: bird species, positive effects, railway network, habitat heterogeneity

Összefoglalás A közlekedési infrastruktúra - például a vasút- és úthálózat - legtöbbször negatív hatással van a természetes környezetre. Ezeknek az ember alkotta létesítményeknek a faunákra nézve azonban pozitív hozadéka is lehet, melyet e vizsgálat is igazol. A kutatás során azt tanulmányozták, hogy az egyes madárfajok hogyan használják a vasútállomásokat - mint az ember által létrehozott struktúrákat - egy városi-vidéki gradiens mentén, az indiai Kolkata külvárosában. A teljes vizsgálati időszak alatt kilenc különböző vasútállomás mentén összesen 12 rendbe tartozó 43 madárfajt és 26 családot figyeltek meg. A fajösszetételben az énekesmadár-alakúak rendje (Passeriformes) dominált 18 különböző fajjal, 11 különböző családból. A városi vasútállomásokon összesen 23 madárfajt (22 nemzetség és 14 család), az elővárosi vasútállomásokon összesen 35 madárfajt (32 nemzetség és 22 család) dokumentáltak. A vidéki régió vasútállomásai mutatták a legmagasabb fajszámot, a családokat tekintve pedig a legnagyobb abundancia értéket: ebben a térségben összesen 36 madárfajt észleltek 32 nemzetségből és 23 családból. Az elővárosi és a vidéki vasútállomások fajösszetételükben hasonlóbbak voltak. A helyszínektől függetlenül a teljes vizsgálati időszak alatt az indiai varjú (Corvus splendens) volt az uralkodó faj, amelyet a pásztormejnó (Acridotheres tristis) követett. Körülbelül 18 faj mutatott csökkenő populációs tendenciát a globális populáció trendelemzés alapján. Valamennyi vasútállomáson a mindenevők domináltak. A vidéki régiókban nagyobb számban voltak jelen magevők, a városi régiókból hiányoztak a nektárevők. Nyilvánvalóvá vált, hogy a vasútállomások pozitív hatást gyakorolnak a madárfajok együtteseire, és a kedvező állapot megfelelő környezetvédelmi tervezéssel és környezetgazdálkodással - beleértve a városok zöldítését is - hosszú távon is fenntartható lehet.

Kulcsszavak: madárfajok, pozitív hatás, vasúthálózat, élőhely-heterogenitás 


\footnotetext{
${ }^{1}$ Department of Zoology, University of Calcutta, 35 Ballygunge Circular Road, Kolkata 700019. India

${ }^{2}$ Department of Zoology, The University of Burdwan, Golapbag, Burdwan 713104. India

*corresponding author, e-mail: gautamaditya2001@gmail.com
}

\section{Introduction}

Birds are one of the most suitable species to monitor the short and the long-term environmental changes (Koskimies 1989, Bibby 1999, Khan \& Naher 2009). Use of birds as indicators for habitat conditions (Canterbury et al. 2000, Browder et al. 2002, Vallecilo et al. 2016), including forests (Pain et al. 2004, Venier \& Pearce 2004, Aich \& Mukhopadhyay 2008, Chatterjee et al. 2014), agro-ecosystems (Dhindsa \& Saini 1994, Borad et al. 2001, Basavarajappa 2006, Sundar \& Kittur 2013, Hossain \& Aditya 2016) and aquatic bodies (Kumar et al. 2006, Kumar \& Gupta 2013), biological diversity (Gregory et al. 2003, Fraixedas et al. 2020) and urbanization (Pollack et al. 2017) are well-recognized, apart from the application of birds as flagship species in conserving diversity (Williams et al. 2000) and enhancing tourism (Veríssimo et al. 2009). Due to an increasing appreciation of the ecosystem services provided by the birds (Whelan et al. 2008, Şekercioğlu 2012a, 2012b), the focus for the monitoring, conservation and ecological studies on birds are continued at different biogeographical context (Bradford et al. 1998, Browder et al. 2002, Lee et al. 2004, Sundar \& Kittur 2013, Hossain \& Aditya 2016). Initiation for the conservation strategies for the birds requires idea about the assemblage pattern at varied spatio-temporal scales. This applies particularly for the conservation initiative in the urban landscapes, which offer habitats for the birds in the form of gardens, parks and green lanes (Chamberlain et al. 2009). In comparison to the limited attention given to human-dominated urban biodiversity earlier (Melles et al. 2003), in the last few decades, there is an increasing awareness about the faunal biodiversity including birds of urban landscapes and their importance in bio-monitoring and conservation perspectives.

Urban landscapes differ substantially and extensively from natural and semi-natural habitats (Marzluff 2001, Chace \& Walsh 2006), in terms of food resources, predator communities (Haskell et al. 2001, Sorace 2002), weather conditions (Haggard 1990), and pollution disturbance (Eeva et al. 2000). As a consequence, the bird assemblages vary considerably revealed through studiesfrom urban areas of India (Sengupta et al. 2014, Kale et al. 2018a, 2018b, Pal et al. 2019) and elsewhere (Chace \& Walsh 2006, Chamberlain et al. 2009). In many instances, the rapid urbanization in association with infrastructural revolution leads to extensive modification of natural landscapes that eventually results in a profound restructuring of the preferred habitats of birds (Blair 1996, Chamberlain et al. 2009, Morelli et al. 2014). As a result, an alteration in the bird species assemblages may be observed along the urbanization gradient with varying levels of the habitat conditions and the degree of disturbances (Gering \& Blair 1999, Kale et al. 2018a, 2018b, Rodrigues et al. 2018, Filloy et al. 2019, Pal et al. 2019). Although the effects of urbanization on the environmental processes are usually complex and poorly understood, the birds can be considered as bio-monitoring tool to retrieve the consequences on human and wildlife biota (Chace \& Walsh 2006, Pollack et al. 2017). 
An inherent aspect of the urbanization is the increased transportation network in the form of metallic roads and railroads that pose a complete different extent of challenges on the biota (Gilbert 2012), including birds (Beissinger \& Osborne 1982, Benítez-López et al. 2010, Morelli et al. 2014). In addition to the continuous increase of global human population, the improvement in transportation network is now getting more compulsory, therefore, the enlargement of urban regions and associated railway networks is indisputable. Thus, proper knowledge and understanding of the relationships between avian species and railway networks and associated human structures should be beneficial for conservation-focused landscape management (Morelli et al. 2014, Wiącek et al. 2015). Railways play an essential role in the global transportation service and currently being promoted by various governments because of their superior economic and environmental advantages relative to other transportation means. Apart from economic benefits, railways also present several environmental advantages in contrast to roads, such as lower pollution and reduced land occupancy (Profillidis 2006, Pereira et al. 2012), resulting in the growth of the railway network, globally and emergence of the research on railway ecology (Borda-de-Água et al. 2017). As a consequence of an increased railway network, the wildlife faces severe challenges in the form of habitat loss, habitat fragmentation, anthropogenic interference, and audio-visual disorder, mortality by collisions, barrier effect, and chemical pollution (Morelli et al. 2014, Borda-de-Água et al. 2017). Thus, the railway network emerges as a prospective and perceived threat to the wildlife biota, including birds. However, the potential positive consequences of the railway network as well as the rail-associated construction structures on birds are increasingly appreciated (Li et al. 2010, van der Ree et al. 2011, Morelli et al. 2014, Wiącek et al. 2015, 2019, 2020, Kaiser-Bonk et al. 2019). Some of the plausible positive effects of rail-associated structures includes the marginal vegetation along railways (bridges, shrubs etc.) may provide nesting sites for several species of birds (Wiącek et al. 2015, 2019, 2020, Kaiser-Bonk et al. 2019), high structures like electricity posts and cables are extensively utilized by many passerine species for perching (DeGregorio et al. 2014, Morelli et al. 2014), singing and relaxing. The railway platform can also act as a good foraging ground for several species of birds and lastly, some birds utilize railway tracks as a resting site as it becomes heated rapidly during the day and thereby providing protection from cold and windy situations during winter (Morelli et al. 2014, Wiącek et al. 2015). All these factors render a positive effect on birds and possibly the reason why they are found in higher aggregations near rail-associated structures than in the deeper forest (Wiącek et al. 2015, 2019, 2020).

In view of the positive effects of the railway network on the bird species assemblages $(\mathrm{Li}$ et al. 2010, Morelli et al. 2014, Wiącek et al. 2015, 2019, 2020, Kaiser-Bonk et al. 2019), it would be worthy to evaluate for any variations in the effect against the urban-rural gradient. As a mode of transport, the railway network extends beyond the urban landscapes and more commonly dissects the rural areas including forests. A gradient of urbanization results in a differntial level of bird species richness, including variations in the foraging guilds and similarity in species composition (Kale et al. 2018a, 2018b, Pal et al. 2019), which may also apply for the railway network connecting urban and rural destinations. In order to justify this proposition, a pioneer attempt was made to explore the bird species diversity in 
railway stations of urban, semi-urban, and rural landscapes of West Bengal, India. The primary objectives of the study were (1) to make a checklist of the birds observed in railway stations, (2) to evaluate the species diversity and the functional diversity of the birds in the railway stations along an urban rural gradient and (3) to evaluate species specific differences in abundance in the railway stations along an urban rural gradient. Elucidation of the species specific benefits derived from the railway network will enhance the sustenance of diversity (Grimmett et al. 2016) and the ecosystem services (Whelan et al. 2008, Şekercioğlu 2012a, 2012b) of birds, especially in an Indian context. Apart from substantiating the urbanization effect, the results will validate the role of railway stations in supporting bird assemblages and thus, prospects in conservation management.

\section{Materials and methods}

\section{Study area}

Our present study on avian biodiversity was carried out between March and May in 2019 in railway stations in West Bengal, India. To carry out the study, nine railway stations were selected randomly in between Howrah railway junction and Barddhaman railway junction (Figure 1) of the Eastern Railways section of Indian Railways. Three railway stations [Ta-

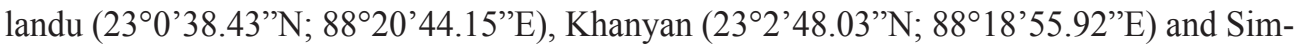
lagarh $\left(23^{\circ} 5^{\prime} 46.95^{\prime \prime} \mathrm{N} ; 88^{\circ} 13^{\prime} 53.38^{\prime \prime} \mathrm{E}\right)$ ] were located in rural areas (R), three stations [Baidy-

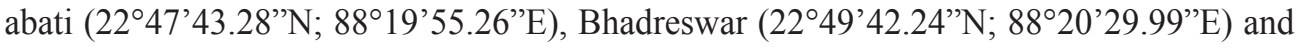
Mankundu (22 $50^{\prime} 48.99^{\prime \prime} \mathrm{N}$; $\left.88^{\circ} 20^{\prime} 48.90^{\prime} \mathrm{E}\right)$ ] were selected from suburban areas (SU), and the remaining three railway stations [Liluah $\left(22^{\circ} 37^{\prime} 14.88^{\prime \prime} \mathrm{N} ; 88^{\circ} 20^{\prime} 21.84^{\prime \prime} \mathrm{E}\right)$, Belur $\left(22^{\circ} 38^{\prime} 8.88^{\prime \prime} \mathrm{N} ; 88^{\circ} 20^{\prime} 23.27^{\prime \prime} \mathrm{E}\right)$ and Uttarpara $\left.\left(22^{\circ} 40^{\prime} 2.99^{\prime \prime} \mathrm{N} ; 88^{\circ} 20^{\prime} 28.33^{\prime \prime} \mathrm{E}\right)\right]$ were chosen from urban areas (U). The categorization of urban, suburban, and rural areas was designated based on population size and density. The surroundings of $U$ areas were enriched mainly with large buildings and small factories while the SU areas were surrounded by relatively smaller houses, discrete vegetation as well as small water bodies at the vicinity. The $\mathrm{R}$ areas were encircled mainly by agricultural lands and jungles. All of these nine stations covered three districts (Howrah, Hooghly, and Purba Bardhaman) of West Bengal, India. The average temperature in the studied areas varied from $35-45^{\circ} \mathrm{C}$ in summer (March to May), relative humidity lied between 50 and $75 \%$, depending on weather conditions with an average annual rainfall of $150 \mathrm{~mm}$ for the concerned area.

\section{Methodology}

The birds were observed and counted in sampling sites for three consecutive months from March to May in 2019. Each site was intensely surveyed twice every month at an interval of two weeks. Adopting line transect method and maintaining constant transect length the surveys were carried out in each selected railway station in the morning time (between 6:00 $\mathrm{AM}$ to 9:00 $\mathrm{AM}$ ) and in the afternoon (from 3:00 PM to 6:00 PM) depending on the 

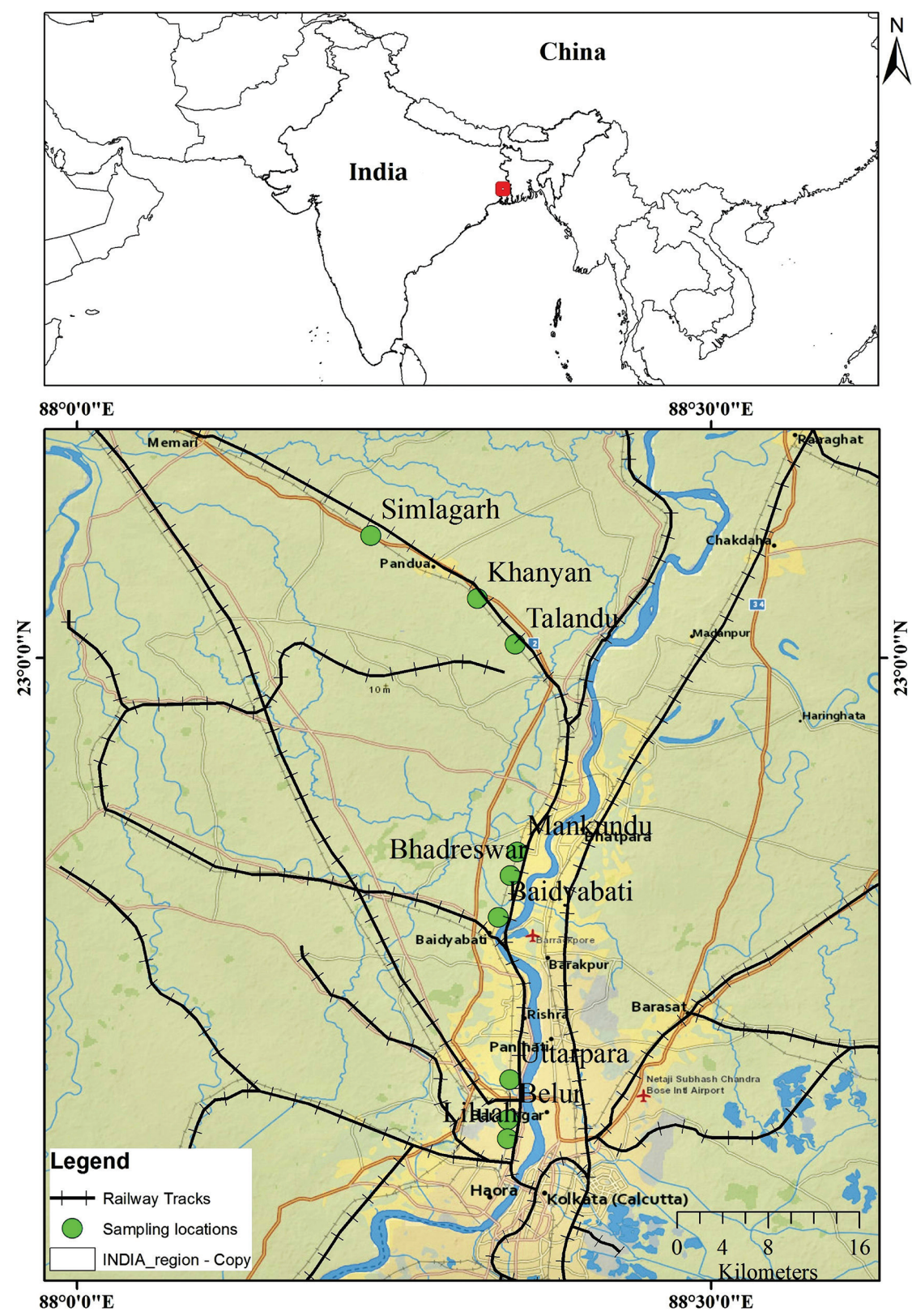

Figure 1. Map of India with the study areas including nine railway stations in West Bengalbeing highlighted. Three stations (Talandu, Khanyan and Simlagarh) were chosen from rural areas (R), three stations (Baidyabati, Bhadreswar and Mankundu) were from suburban areas (SU) and other three railway stations (Liluah, Belur and Uttarpara) were taken from urban areas (U)

1. ábra A vizsgálati terület térképe, 9 nyugat-bengáliai vasútállomás megjelölésével. Három állomás (Talandu, Khanyan és Simlagarh) vidéki (R), három állomás (Baidyabati, Bhadreswar és Mankundu) elővárosi (SU) és három állomás (Liluah, Belur és Uttarpara) városi (U) területről lett választva 
day length when the birds were found to be more active (Buckland et al. 1993, Bibby et al. 2000). The starting point and the direction of transects were often arbitrary. Each survey site was visited six times throughout the study period, three times in the morning time and three times in the afternoon. Cloudy and overcast days were strictly avoided for a field visit. The birds were observed either by unaided eyes or by binocular (Olympus 7x21 PS III) depending upon distance and photographs were captured by Nikon P900 for documentation of the avifauna. Based on observations and captured photographs, birds were identified (Ali 1996, Grimmett et al. 2016) and recorded for tabulation and statistical analysis. In some cases, birds' calls were used as an identifying key. The taxonomic categorization of bird species was made by following Praveen et al. 2016. During the survey period, the foraging behaviours, nesting and resting positions, areas of displaying and singing as well as their overall activities were observed.

\section{Data analysis}

The information about the status of global population trends for each observed bird species was collected from the IUCN Red List (del Hoyo et al. 1996). Foraging guilds were determined by examining their feeding habitat and categorized into six feeding guilds i.e. carnivore (Car), omnivore (Omn), nectarivore (Nect), granivore (Gran), insectivore (Ins) and frugivore (Frug) (Ali \& Ripley 1980, Hutto 1986). To obtain the diversity indices of bird abundance, the data taken from each study site from three areas (U, SU and R) were analyzed separately by using Biodiversity Pro software (McAleece et al. 1997, Biodiversity Professional; Scottish Association for Marine Science and the Natural History Museum, London, UK). Species richness (S) was calculated by summing the number of different species present in that area (Mukherjee et al. 2015, Issa 2019). Diversity of species was represented by calculating Shannon diversity index $\left[\mathrm{H}^{\prime}=-\sum\left(\mathrm{P}_{\mathrm{i}} \ln \mathrm{P}_{\mathrm{i}}\right)\right]$, Simpson's Index of diversity $\left[(1-\mathrm{D})=1-\frac{\sum \mathrm{n}(\mathrm{n}-1)}{\mathrm{N}(\mathrm{N}-1)}\right]$, where $\mathrm{P}_{\mathrm{i}}$ is the proportion of total samples belonging to the $i^{\text {th }}$ species, $\mathrm{n}$ is the total number of birds belongs to a particular species and $\mathrm{N}$ includes the total number of birds of all species (Magurran 1988). To compare the similarity of population size of each bird species in an area, the evenness $\left[\mathrm{J}=\mathrm{H}^{\prime} / \mathrm{H}_{\max }\right]$ was calculated. Fisher's alpha $(\alpha)$ is a parametric diversity index was estimated to analyse diversity within the population. The Margalef's richness was estimated as $\left[\mathrm{D}_{\mathrm{Mg}}=(\mathrm{S}-1) / \mathrm{ln} \mathrm{N}\right]$, (Margalef 1958), where $\mathrm{S}$ is the total number of avian species and $\mathrm{N}$ is the total number of individuals. The relationship among species richness $(\mathrm{S})$, information $(\mathrm{H})$, and evenness $(\mathrm{J})$ in the samples was made by SHE analysis (Buzas \& Hayek 1998). The proximity and similarity of avifaunal community structures of three different habitats (U, SU and R), in terms of species composition was estimated by the Jaccard coefficient $\left(\mathrm{Jc}=\frac{\mathrm{M}}{\mathrm{M}+\mathrm{N}}\right)$ and Sorensen coefficient $\left(\mathrm{Sc}=\frac{2 \times \mathrm{C}}{\mathrm{A}+\mathrm{B}}\right)$, where $\mathrm{M}$ denotes the number of common species between communities, $\mathrm{N}$ is the total number of unique species present in both communities, $\mathrm{C}$ is the common species between two habitats, whereas the components $\mathrm{A}$ and $\mathrm{B}$ are the number of bird species at two different habitats proposed to be compared for similarity (Ludwig \& Reynolds 1988, Krebs 1999). Agglomerative hierarchical clustering (AHC) was carried out based on the Pearson's similarity coefficient of habitat types and avian species richness associated to these habitats. To comment 
on the variation in abundance of different species along the urban gradients, a mixed model ANOVA was performed with the species as the repeated factors and the urban, suburban and rural regions as the fixed factors. In order to specify if there were any difference in their abundance related to time of the day variation, ANOVA was performed (Zar 1999). All abundance data were presented as mean $\pm \mathrm{SE}$ and significance were tested at $\mathrm{p}<0.05$. Diversity index, species richness, evenness and associated analysis were performed by using Biodiversity Pro (2.0) software. The statistical analyses were performed following Zar (1999) using the XLSTAT software (Addinsoft 2010).

\section{Result}

\section{Bird species records and their relative abundance}

During the entire study along the nine different railway stations irrespective of urban to rural gradient, a total of 43 bird species belong to 12 orders and 26 families were observed (Table 1). Among the recorded birds, the order Passeriformes was predominant on its species composition having 18 different species from 11 different families. In urban railway stations (U), a total of 23 bird species belongs to 22 genera and 14 families were observed. The maximum number of bird species recorded in $\mathrm{U}$ sites were under family Columbidae with 4 species (17.40\%) followed by Sturnidae with 3 species (13.04\%), Apodidae, Cuculidae, Corvidae, and Ardeidae, each containing 2 species (8.70\% each) and Accipitridae, Cisticolidae, Dicruridae, Passeridae, Pycnonotidae, Phalacrocoracidae, Picidae, Psittaculidae, each with 1 species ( $4.35 \%$ each). In suburban railway stations (SU), a total of 35 bird species belongs to 32 genera and 22 families were documented. The maximum number of species recorded in SU were under the families Columbidae, Corvidae, Sturnidae, and Ardeidae, each having 3 species ( $8.60 \%$ each), followed by Apodidae, Cuculidae, Cisticolidae, Nectariniidae, and Pycnonotidae, with 2 species (5.71\% each) and Accipitridae, Jacanidae, Alcedinidae, Rallidae, Dicruridae, Hirundinidae, Leiothrichidae, Muscicapidae, Oriolidae, Passeridae, Phalacrocoracidae, Megalaimidae and Picidae, each having 1 species $(2.85 \%$ each). Nevertheless, railway stations from rural regions (R) showed the highest number of species and abundance of bird families, where a total of 36 bird species were recorded throughout the study period which belongs to 32 genera and 23 families. In rural regions $(\mathrm{R})$ the maximum number of species listed under families Columbidae, Corvidae, and Sturnidae each of which has 3 species ( $8.3 \%$ each), followed by Apodidae, Alcedinidae, Cuculidae, Nectariniidae, Pycnonotidae, Ardeidae, and Megalaimidae, each with 2 species (5.6\% each), and Anatidae, Rallidae, Cisticolidae, Dicruridae, Leiothrichidae, Muscicapidae, Oriolidae, Passeridae, Ciconiidae, Phalacrocoracidae, Threskiornithidae, Picidae, and Psittaculidae, each having 1 species ( $2.8 \%$ each). The possible variations in the relative abundance of different observed families in three different sites ( $U$, $\mathrm{SU}$ and R) might be due to the alterations in habitat conditions. The relative abundance of bird species (Figure 2) and the abundance of families (Figure 3) were highest in the rural railway stations followed by suburban and urban areas. 


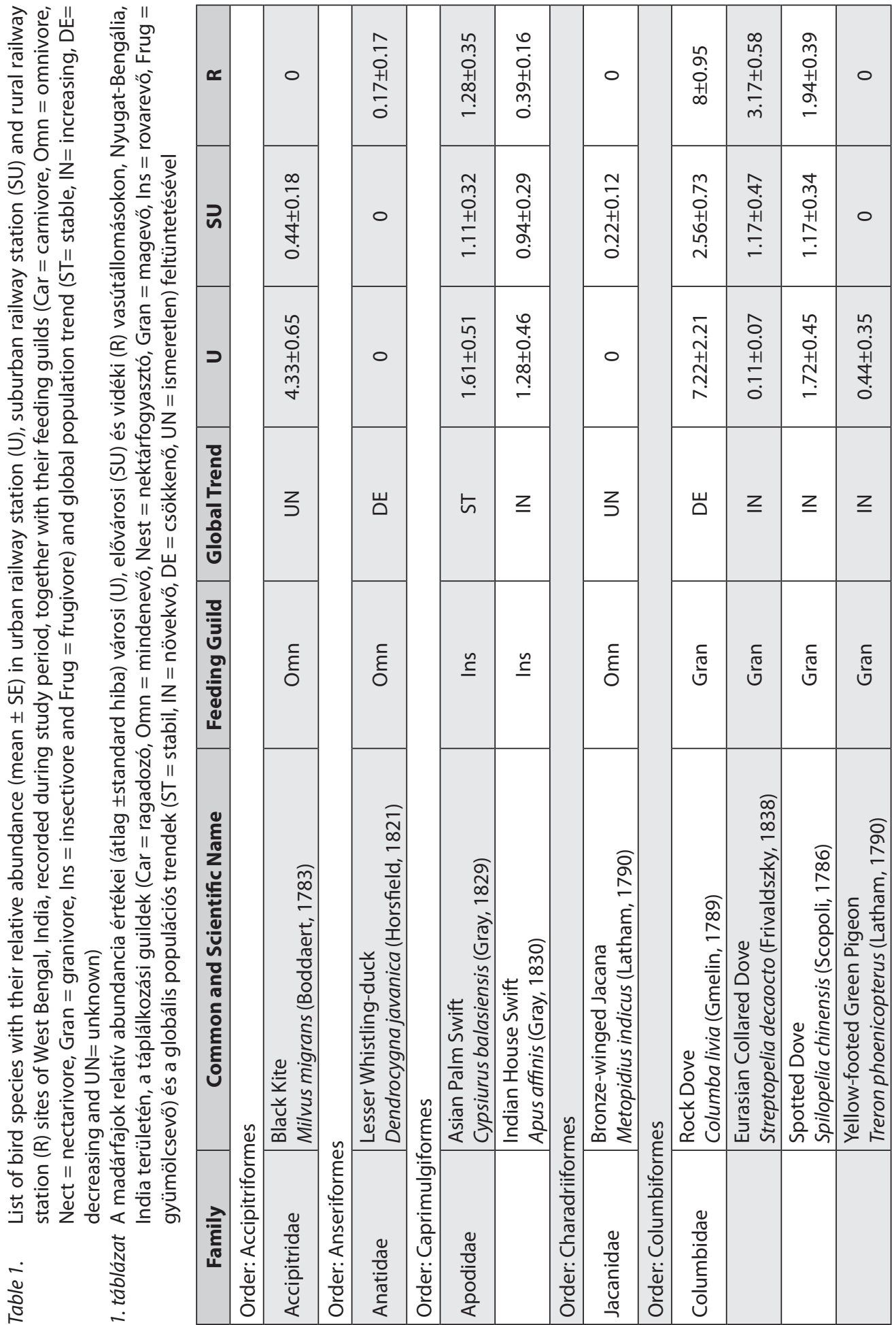




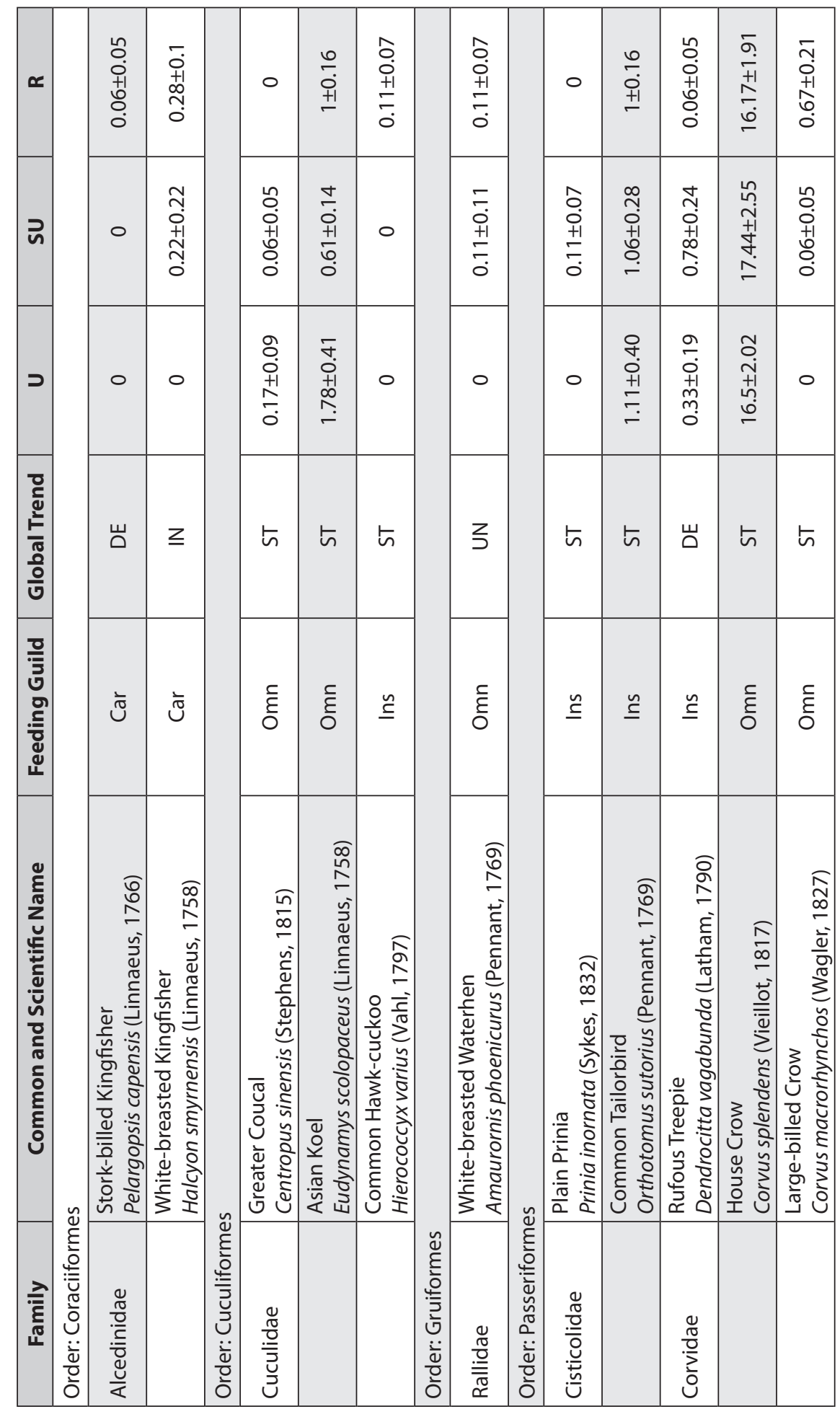




\begin{tabular}{|c|c|c|c|c|c|c|c|c|c|c|c|c|c|c|}
\hline$\propto$ & & 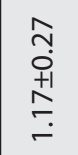 & 0 & 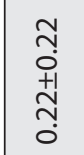 & 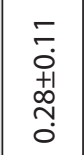 & 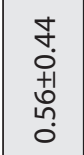 & $\begin{array}{l}\bar{\sigma} \\
\overline{0} \\
+1 \\
\frac{+1}{0}\end{array}$ & $\begin{array}{l}\text { 우 } \\
0 \\
+1 \\
0 \\
0 \\
0\end{array}$ & $\begin{array}{l}\text { fo } \\
\text { o } \\
+1 \\
\stackrel{1}{0} \\
\stackrel{+}{r}\end{array}$ & $\begin{array}{l}\bar{\Xi} \\
0 \\
+1 \\
\frac{1}{0}\end{array}$ & $\begin{array}{l}\mp \\
\overline{+1} \\
+1 \\
0 \\
0\end{array}$ & $\begin{array}{l}\stackrel{p}{m} \\
\stackrel{+1}{+1} \\
m \\
m \\
m \\
m\end{array}$ & 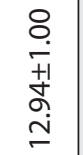 & $\begin{array}{l}\tilde{m} \\
0 \\
+1 \\
+0 \\
\stackrel{0}{0}\end{array}$ \\
\hline ฉ & & $\begin{array}{l}\bar{N} \\
0 \\
+1 \\
0 \\
0 \\
0\end{array}$ & \begin{tabular}{l}
$\forall$ \\
\multirow{0}{*}{} \\
+1 \\
$\forall$ \\
\multirow{0}{*}{}
\end{tabular} & $\begin{array}{l}n \\
m \\
0 \\
+ \\
+1 \\
\infty \\
\infty \\
0\end{array}$ & $\begin{array}{l}\stackrel{\infty}{\check{0}} \\
+1 \\
m \\
m \\
0\end{array}$ & 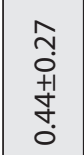 & $\begin{array}{l}9 \\
0 \\
0 \\
+1 \\
\frac{1}{0} \\
0\end{array}$ & $\begin{array}{l}\circ \\
\\
+1 \\
\stackrel{+}{+} \\
0\end{array}$ & 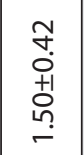 & $\begin{array}{l}\text { ơ } \\
\text { o } \\
+1 \\
\text { ○े }\end{array}$ & 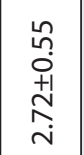 & 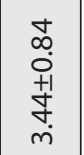 & 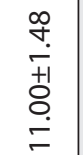 & $\begin{array}{l}\stackrel{m}{0} \\
\stackrel{0}{+} \\
\stackrel{+}{N} \\
0\end{array}$ \\
\hline כ & & $\begin{array}{l}= \\
\dot{0} \\
+1 \\
m \\
m \\
0\end{array}$ & 0 & 0 & 0 & 0 & 0 & 0 & $\begin{array}{l}+ \\
\dot{0} \\
+1 \\
m \\
m \\
0\end{array}$ & 0 & 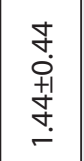 & 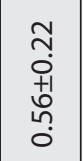 & $\begin{array}{l}\infty \\
o \\
o \\
+1 \\
\text { on } \\
\text { m. } \\
0\end{array}$ & $\begin{array}{l}0 \\
\frac{0}{0} \\
+1 \\
\stackrel{+1}{0} \\
0\end{array}$ \\
\hline $\begin{array}{l}\text { 을 } \\
\text { 인 } \\
\text { 응 }\end{array}$ & & క & 㟔 & 㐫 & $\hbar$ & $\grave{n}$ & 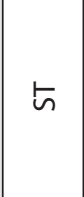 & Ł & 岁 & 㟔 & $\geqq$ & $\geqq$ & $\underline{z}$ & 㟧 \\
\hline 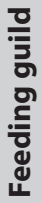 & & 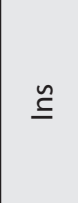 & $\underline{\cong}$ & 气ૃ & $\underline{\subseteq}$ & $\frac{\tilde{u}}{z}$ & $\frac{\breve{U}}{Z}$ & ڤૃ & ڤૃ & ڤૃ & §ૃ & §ૅ & 气ે & §ૃ \\
\hline 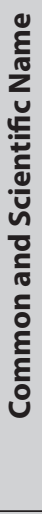 & 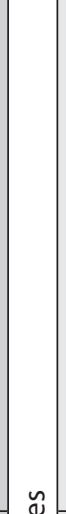 & 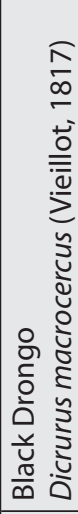 & 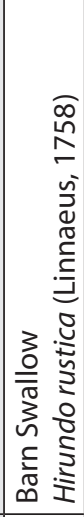 & 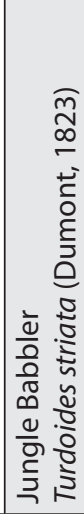 & 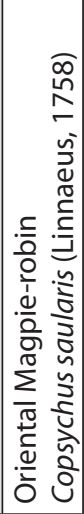 & 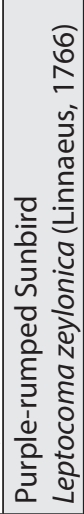 & 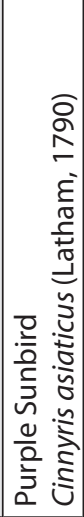 & 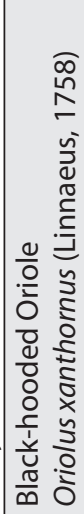 & 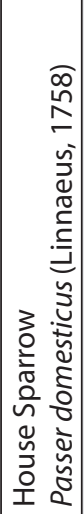 & 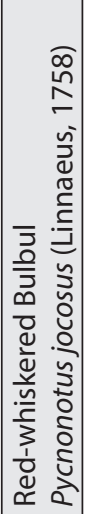 & 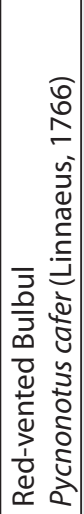 & 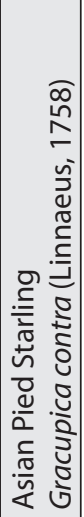 & 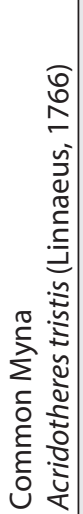 & 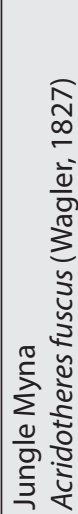 \\
\hline $\begin{array}{l}\overline{\bar{\varepsilon}} \\
\bar{\leftarrow} \\
\stackrel{5}{L}\end{array}$ & 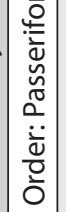 & $\begin{array}{l}\frac{0}{\pi} \\
\frac{\pi}{0} \\
\frac{0}{3} \\
\frac{2}{2} \\
\frac{0}{0}\end{array}$ & 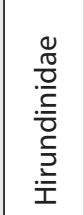 & 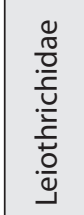 & 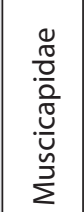 & 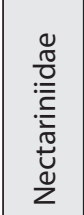 & & $\begin{array}{l}\frac{\pi}{\pi} \\
\frac{\pi}{0} \\
\frac{0}{0} \\
.0 \\
0\end{array}$ & 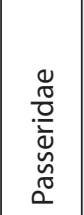 & 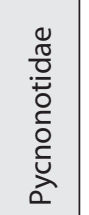 & & 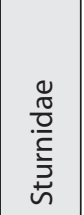 & & \\
\hline
\end{tabular}




\begin{tabular}{|c|c|c|c|c|c|c|c|c|c|c|c|c|c|}
\hline$\simeq$ & & $\begin{array}{l}\text { Ln } \\
0 \\
0 \\
+1 \\
0 \\
0 \\
0\end{array}$ & $\begin{array}{l}\stackrel{N}{\hat{T}} \\
\stackrel{+1}{+1} \\
\text { m. } \\
0\end{array}$ & 0 & $\begin{array}{l}m \\
m \\
o \\
+1 \\
0 \\
\stackrel{n}{1} \\
0\end{array}$ & 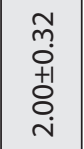 & 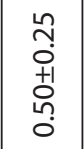 & & $\begin{array}{l}\bar{\Xi} \\
\stackrel{+}{+1} \\
\frac{1}{0}\end{array}$ & 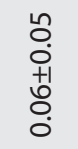 & 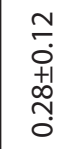 & & 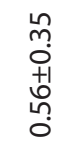 \\
\hline ฉ & & 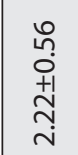 & $\begin{array}{l}\text { 우․ } \\
+1 \\
+1 \\
0 \\
0 \\
0 \\
0\end{array}$ & $\begin{array}{l}\infty \\
0 \\
0 \\
+1 \\
+1 \\
\check{0}\end{array}$ & 0 & $\begin{array}{l}\text { gे } \\
\text { o } \\
+1 \\
\stackrel{1}{0} \\
\text { ஸุ }\end{array}$ & 0 & & 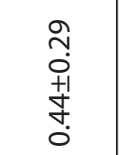 & 0 & $\begin{array}{l}\bar{\sigma} \\
\dot{0} \\
+1 \\
\frac{1}{0}\end{array}$ & & 0 \\
\hline כ & & $\begin{array}{l}\text { Ln } \\
0 \\
0 \\
+1 \\
0 \\
0 \\
0\end{array}$ & $\begin{array}{l}\text { ᄋ } \\
0 \\
+1 \\
\text { +1 } \\
\stackrel{0}{0}\end{array}$ & 0 & 0 & $\begin{array}{l}\hat{\sigma} \\
\frac{+1}{+1} \\
\text { J } \\
\text { N }\end{array}$ & 0 & & 0 & 0 & $\begin{array}{l}\text { 우 } \\
\circ \\
\circ \\
+1 \\
0 \\
0 \\
0\end{array}$ & & 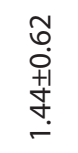 \\
\hline 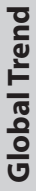 & & 3 & $\underline{z}$ & $\underline{z}$ & క & ろ & 㟔 & & $\underline{z}$ & ら & 更 & & $\geqq$ \\
\hline 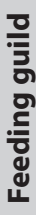 & & ن & $\cup^{\bar{\pi}}$ & ن্ত & U் & U & 心 & & $\frac{9}{\frac{9}{4}}$ & 온 & $\underline{\cong}$ & & $\frac{\text { 온 }}{\frac{1}{2}}$ \\
\hline 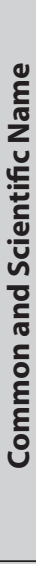 & $\tilde{\varrho}$ & 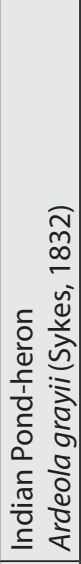 & 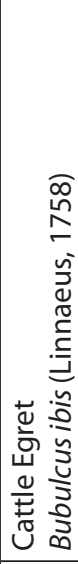 & 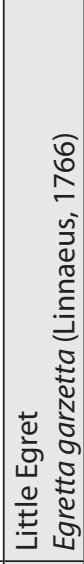 & 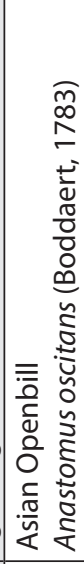 & 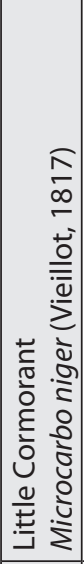 & 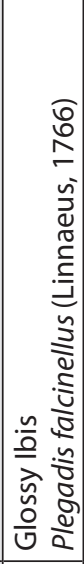 & & 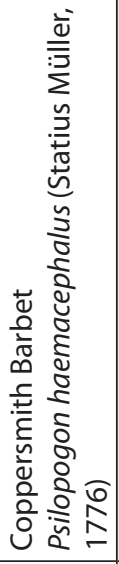 & 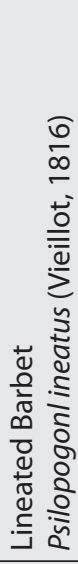 & 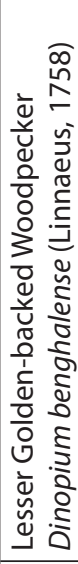 & $\simeq$ & 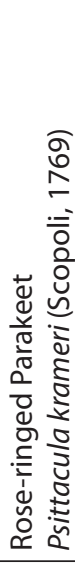 \\
\hline 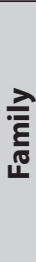 & 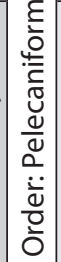 & $\begin{array}{l}\frac{0}{0} \\
\frac{0}{0} \\
\frac{0}{0} \\
\frac{0}{\alpha}\end{array}$ & & & 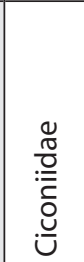 & 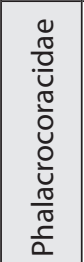 & 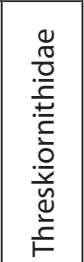 & 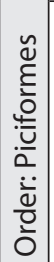 & 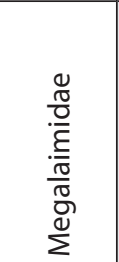 & & $\frac{\mathscr{U}}{\frac{\pi}{\underline{U}}}$ & 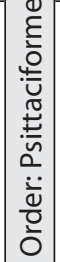 & 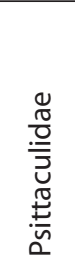 \\
\hline
\end{tabular}




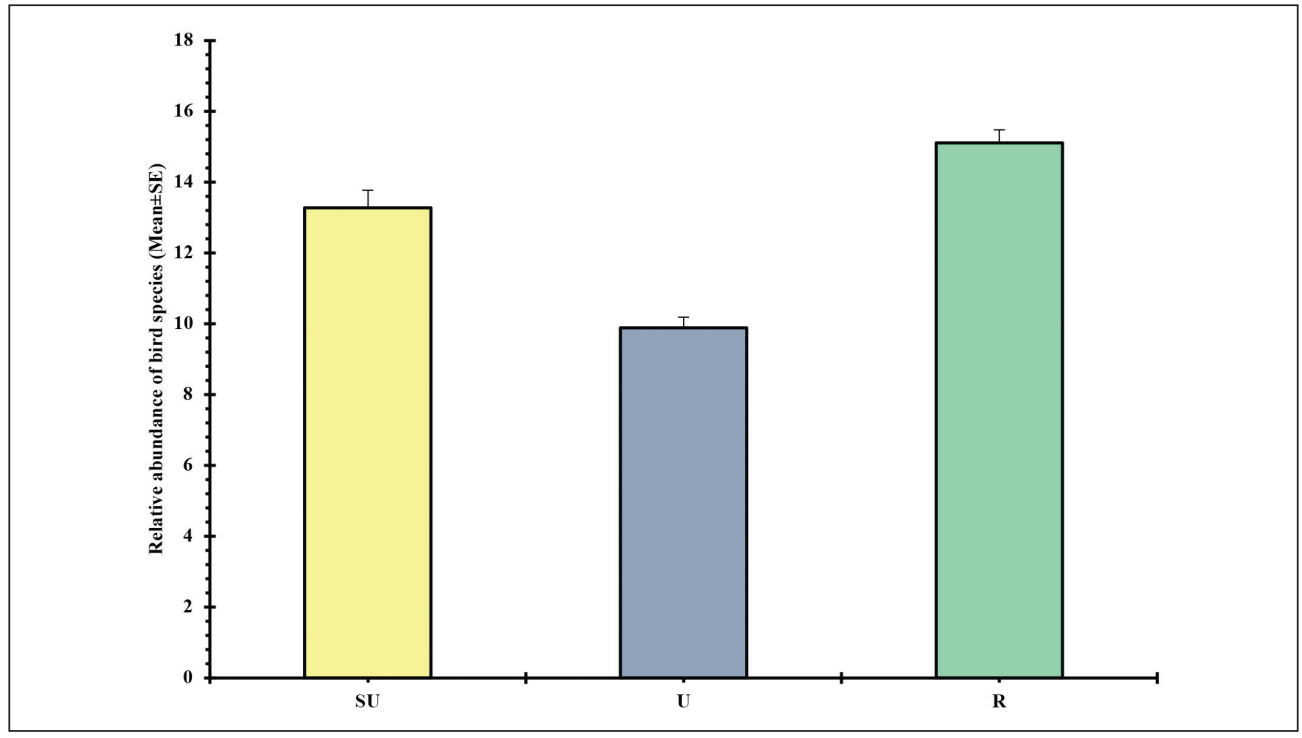

Figure 2. Relative abundance of bird species recorded from three different sites ( $S U, U$ and R)

2. ábra A madárfajok relatív abundanciája három különböző területtípusban (elővárosi (SU), városi (U) és a vidéki (R))

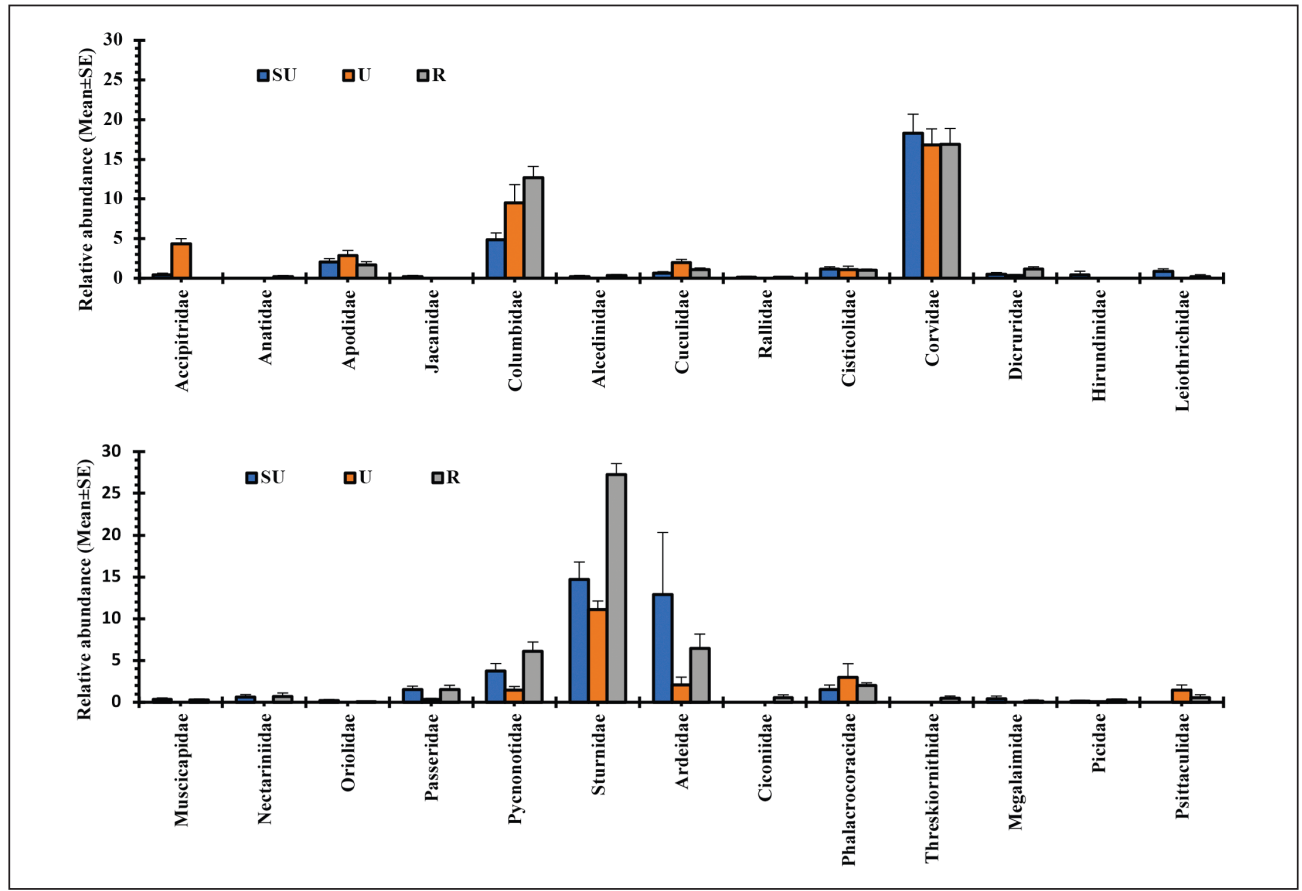

Figure 3. The relative abundance of the different bird family in the suburban (SU), urban (U) and rural (R) station areas sampled during the study period

3. ábra A különböző madárcsaládok relatív abundanciája az elővárosi (SU), városi (U) és a vidéki (R) állomásokon 


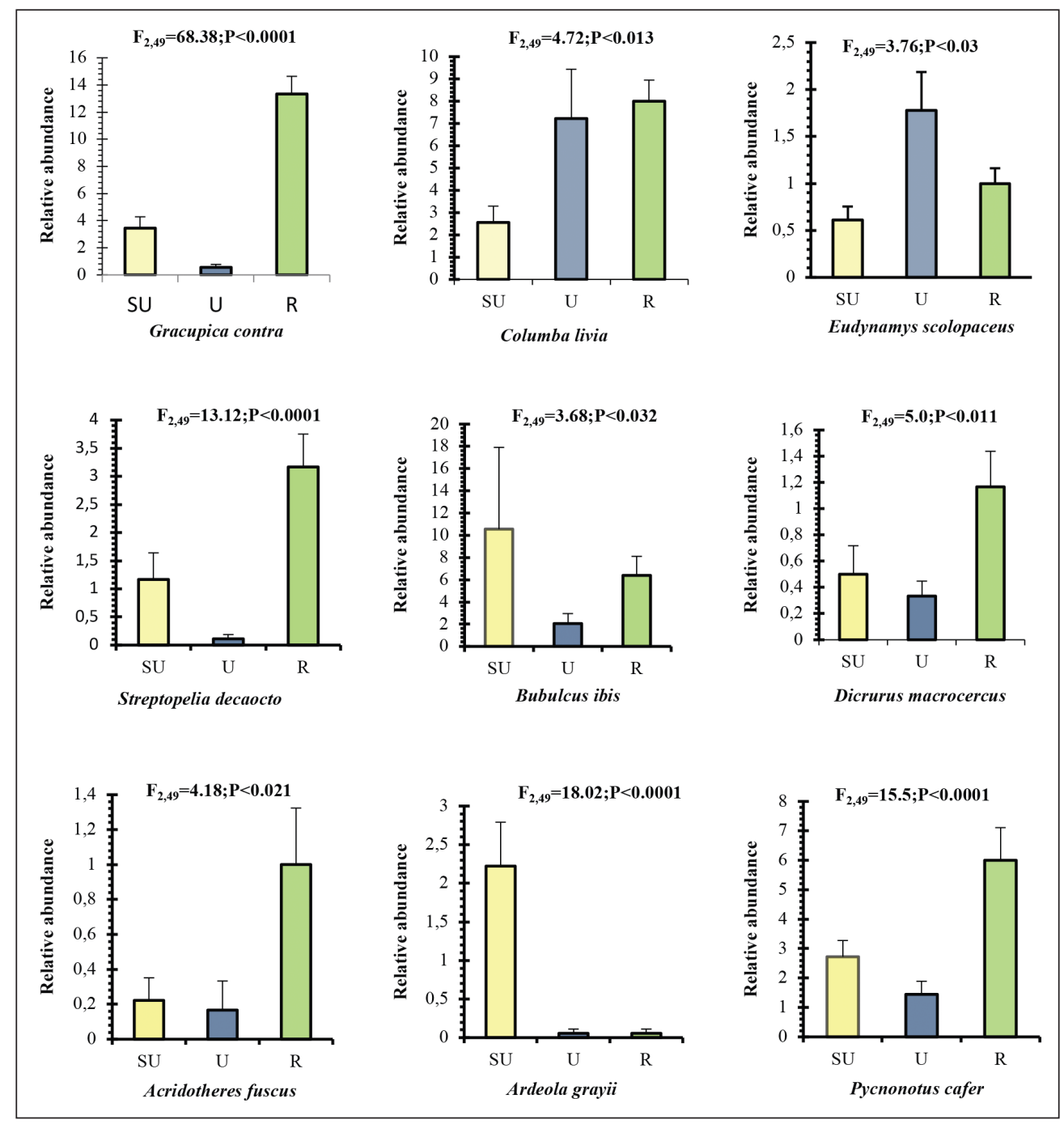

Figure 4. Selected bird species, which were available in each habitat $(\mathrm{SU}=$ suburban, $\mathrm{U}=$ urban and $R=$ rural) and are known to differ significantly $(P<0.05)$ in their relative abundance

4.ábra A relatív abundanciájukban jelentősen különböző madárfajok összehasonlítása terület típusonként (elővárosi (SU), városi (U) és a vidéki (R))

During the entire study period, House Crow (Corvus splendens) was the most predominant species in all railway stations irrespective of three different landscapes with a relative abundance (mean $\pm \mathrm{SE}$ ) of $16.5 \pm 2.0$ for $\mathrm{U}, 17.4 \pm 2.5$ for SU and $16.2 \pm 1.9$ for $\mathrm{R}$ areas. Compared to House Crows, in $\mathrm{U}$ the abundance of the total number of species was higher for Common Myna (Acridotheres tristis) (10.4 \pm 0.9$)$ and lowest for Ardeola grayii and Dinopium benghalense ( $0.06 \pm 0.1$ for each). In addition to that, Acridotheres tristis was also

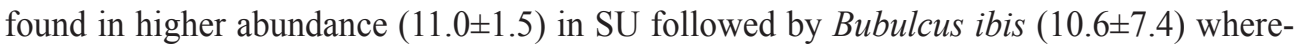
as, minimum relative abundance recorded from species Centropus sinensis and Corvus 
macrorhynchos with $0.1 \pm 0.1$, for each. In addition to Corvus splendens, the relative abundance of Gracupica contra and Acridotheres tristis were also higher in $\mathrm{R}$ with an abundance of $13.3 \pm 1.3$ and $12.9 \pm 1.0$, respectively, although, least relative abundance recorded from species Pelargopsis capensis, Dendrocitta vagabunda, Oriolus xanthornus, Ardeola grayii, Psilopogon lineatus each which mean abundance value of $0.06 \pm 0.05$.

Species based relative abundance in three different areas (Figure 4) were analyzed. Significant difference $\left(\mathrm{F}_{2,49}=68.38, \mathrm{P}<0.0001\right)$ was found on the distribution of Asian Pied Starling (Gracupica contra) in higher number in rural railway areas and in lowest number in urban railway stations. Rock Dove (Columba livia) was found to be more abundant in Rural railway stations and relatively moderate number in urban railway stations and lowest number in suburban railway stations $\left(\mathrm{F}_{2.49}=4.72, \mathrm{P}=0.013\right)$. Abundance of Red-vented Bulbul (Pycnonotus cafer) was found in higher number in rural railway stations and lowest in urban railway station $\left(\mathrm{F}_{2,49}=15.5, \mathrm{P}<0.0001\right)$, as well as Jungle Myna (Acridotheres fuscus) was also found in higher number in rural railway stations and lowest in urban railway stations $\left(\mathrm{F}_{2,49}=4.18, \mathrm{P}=0.021\right)$. Eurasian Collared Dove (Streptopelia decaocto) $\left(\mathrm{F}_{2,49}=13.12, \mathrm{P}<0.0001\right)$ and Black Drongo (Dicrurus macrocercus) $\left(\mathrm{F}_{2,49}=5.0, \mathrm{P}=0.011\right)$ were found in higher number in the rural area and relative moderate number in semi urban area and were found lowest in urban area. Significant difference in the abundance $\left(\mathrm{F}_{2,49}=3.76, \mathrm{P}=0.03\right)$ of Asian Koel (Eudynamys scolopaceus) was found in higher number in urban area and lowest in the semi-urban area. Significant difference in the abundance of Cattle Egret (Bubulcus ibis) $\left(\mathrm{F}_{2,49}=3.68, \mathrm{P}=0.032\right)$, Indian Pond-Heron (Ardeola grayii) $\left(\mathrm{F}_{2,49}=18.02, \mathrm{P}<0.0001\right)$, Rufous Treepie (Dendrocitta vagabunda) $\left(\mathrm{F}_{2,49}=4.34, \mathrm{P}=0.018\right)$ were also noticed along the urban gradient with their higher availability in suburban railway station areas.Considering the time of surveys that were carried out in the morning (6:00 AM to 9:00 AM) and in the afternoon (3:00 PM to 6:00 PM), depending on the day length when birds were found to be most active, it was reported that expect of two species (Cypsiurus balasiensis, Halcyon smyrnensis), other species did not show significant differences $(\mathrm{P}>0.05)$ in both morning and afternoon time irrespective of urban-rural gradient (Figure 5).The number of Asian Palm Swift $\left(\mathrm{F}_{1,52}=4.7, \quad \mathrm{P}<0.05\right)$ and White Throated Kingfisher $\left(\mathrm{F}_{1,52}=5.49, \mathrm{P}<0.05\right)$ are significantly differ in their abundance in

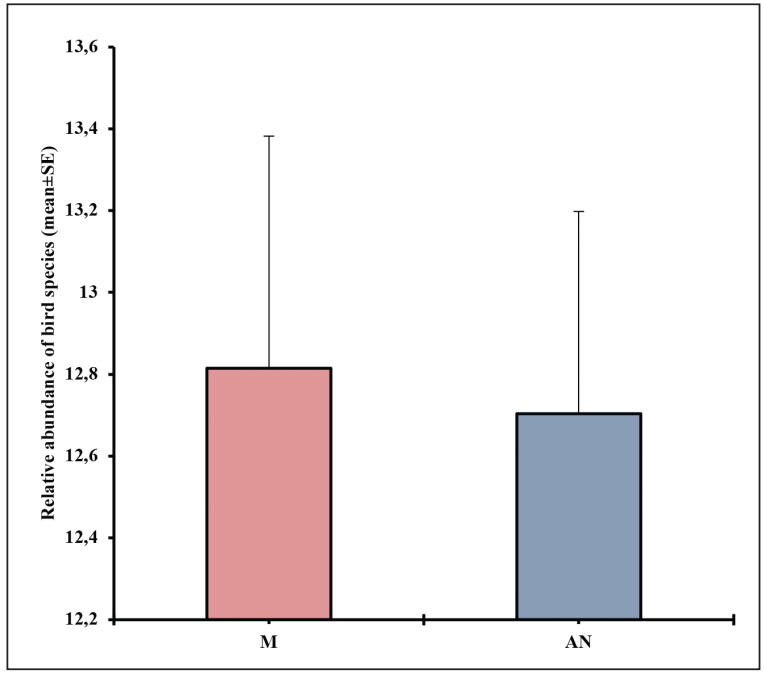

Figure 5. Relative abundance of bird species observed during different sampling time ( $M=$ Morning, AN = Afternoon) from all stations irrespective of rural-urban gradient

5. ábra A reggeli (M) és délutáni (AN) mintavételi órákban megfigyelt relatív abundancia értékek 


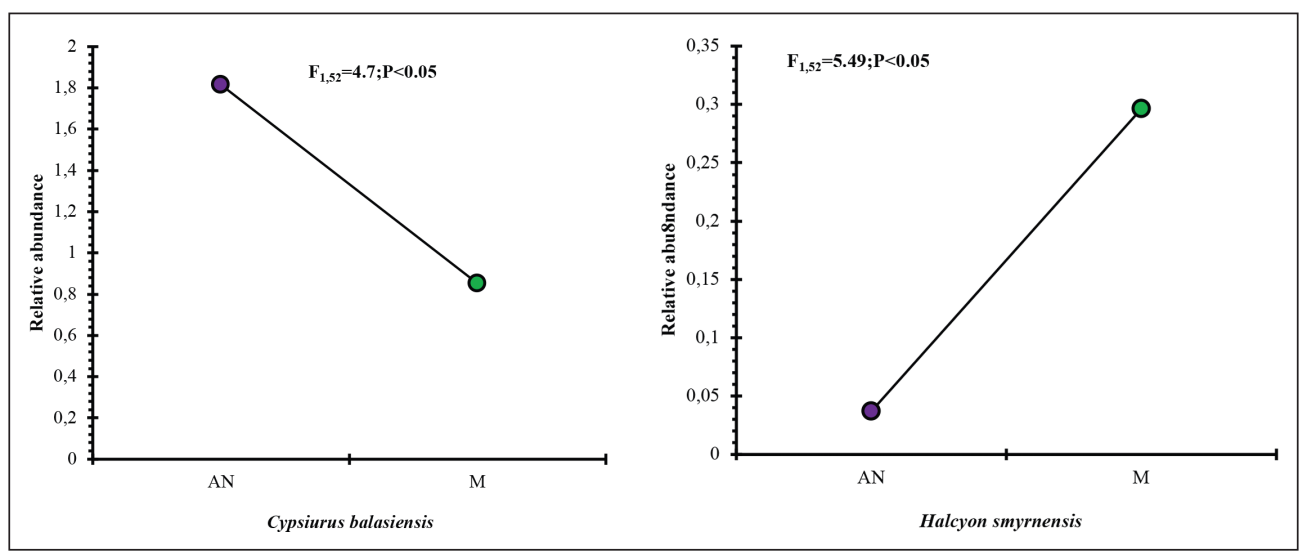

Figure 6. Relative abundance of selected bird species differ significantly $(\mathrm{P}<0.05)$ in different sampling time ( $\mathrm{M}=$ Morning, $\mathrm{AN}=$ Afternoon)

6. ábra A mintavételi időszakokban ( $M=$ reggel, $\mathrm{AN}=$ délután) jelentősen eltérő relatív abundanciájú madárfajok összehasonlítása

between the two times (Figure 6). Asian Palm Swift (Cypsiurus balasiensis) is more frequent in number at afternoon than in morning whereas the White Throated Kingfisher (Halcyon smyrnensis) is more frequent in morning times $(\mathrm{P}=0.023)$.

\section{Global population trends of the recorded species}

By analysing the global population trend it was noted that the railway stations belong to urban regions includes 6 such species of birds known to follow the stable population trend (ST), 9 increasing (IN), 4 with unknown (UN) and notably other 4 species marked to follow the decreasing (DE) population trend, whereas railway stations from suburban regions with 13 such species that are following the ST population trend, 10 with IN, 6 with UN and other 6 species known follow the DE population trend. On the contrary, railway stations from rural regions include 13 bird species that are known to follow the ST population trend, 10 IN, 5 with UN, and remaining 8 species are following the DE population trend (del Hoyo et al. 1996).

\section{Analysis of diversity indices}

Along the railway stations of urban-rural gradient, the values of diversity indices recorded (Table 2) for bird species as Species richness (S), Shannon diversity index (H'), Simpson's Index of diversity (1-D), evenness (J), Fisher's alpha $(\alpha)$, Margalef's Richness index $\left(\mathrm{D}_{\mathrm{Mg}}\right)$. The highest Shannon diversity index $\left(\mathrm{H}^{\prime}\right)$ of birds recorded in railway stations belongs to rural regions $(2.24 \pm 0.03)$, followed by suburban regions $(2.07 \pm 0.08)$ and urban regions $(1.81 \pm 0.05)$. Species richness $(\mathrm{S})$ was higher in the rural $(15 \pm 0.38)$ and suburban (13.28 \pm 0.49$)$ rail stations than in the urban rail stations $(9.89 \pm 0.30)$, which showed the lowest species richness amongst three sampling regions. The Simpson's 
Table 2. Diversity indices of the bird communities recorded from three different habitats 2. táblázat A három különböző élőhelyet jellemző diverzitási indexek összehasonlítása

\begin{tabular}{|l|c|c|c|}
\hline \multicolumn{1}{|c|}{ Diversity Indices } & SU & U & R \\
\hline Species richness_(S) & $13.28 \pm 0.49$ & $9.89 \pm 0.30$ & $15.00 \pm 0.38$ \\
\hline Simpson's index of diversity_(1-D) & $0.82 \pm 0.02$ & $0.79 \pm 0.01$ & $0.86 \pm 0.01$ \\
\hline Shannon diversity index_( $\left.\mathrm{H}^{\prime}\right)$ & $2.07 \pm 0.08$ & $1.81 \pm 0.05$ & $2.24 \pm 0.03$ \\
\hline Evenness_(J) & $0.80 \pm 0.02$ & $0.79 \pm 0.01$ & $0.83 \pm 0.01$ \\
\hline Fisher_a & $5.64 \pm 0.47$ & $3.59 \pm 0.16$ & $5.54 \pm 0.18$ \\
\hline Margalef_( $\left.\mathrm{D}_{\mathrm{Mg}}\right)$ & $1.73 \pm 0.07$ & $1.28 \pm 0.04$ & $1.93 \pm 0.05$ \\
\hline
\end{tabular}

Index, which measures community diversity found to be highest in the railway stations in rural $(0.86 \pm 0.01)$ regions, followed by the suburban $(0.82 \pm 0.02)$ regions and the urban $(0.79 \pm 0.01)$ regions. Shannon evenness $(\mathrm{J})$ is the ratio of $\mathrm{H}^{\prime}$ to $\mathrm{H}_{\text {max }}$, a measure of species evenness found higher in the railway stations that come under rural $(0.83 \pm 0.01)$ and suburban $(0.8 \pm 0.02)$ regions as compared to urban $(0.79 \pm 0.01)$ regions. The results revealed that railway stations from rural and suburban regions were highly species-rich as compared to urban regions and further demonstrate that from rural to urban matrices, species diversity and richness decreases, showing influences of urbanization on the avian community. Fisher's alpha $(\alpha)$ is a parametric diversity index to estimate the diversity within the population found to be highest in the suburban $(5.64 \pm 0.47)$ railway stations, followed by the rural $(5.54 \pm 0.18)$ and urban $(3.59 \pm 0.16)$ railway stations. The Margalef's Richness index $\left(\mathrm{D}_{\mathrm{Mg}}\right)$, which was used as another measure of species richness also is the highest in rural (1.93 \pm 0.05$)$ sampling sites than that of the suburban $(1.73 \pm 0.07)$ and urban (1.28 \pm 0.04$)$ sites. As revealed through the results of SHE analysis (Figure 7), the relationship among $\mathrm{S}$ (species richness), $\mathrm{H}$ (information), and $\mathrm{E}$ (evenness) in the samples can interpret well and represents higher species richness in the railway stations from the rural and suburban regions than in the urban regions. In addition to the species abundance in samples as a variable for comparison, the rank abundance curves also generated for rail stations belonging to urban-rural gradient (Figure 8), where the species count plotted in descending order for all the species and found to be highest in rural railways, followed by suburban and urban railway station areas. The railway stations from the suburban and rural landscapes were the regions with the most similar species composition. The Jaccard and Sorensen similarity coefficients for these two sampling sites were 0.69 and 0.817 , respectively, and were the highest. In contrast, the rural and urban rail stations had Jaccard and Sorensen similarity coefficients of 0.512 and 0.678 respectively, and were the least similar in terms of species composition (Figure 9a). Dendrogram based on the species richness along different railway stations areas of urban, semi-urban and rural landscapes formed two distinct clusters where rural stations and suburban stations clustered together, whereas urban habitat was in a separate cluster (Figure 9b). 


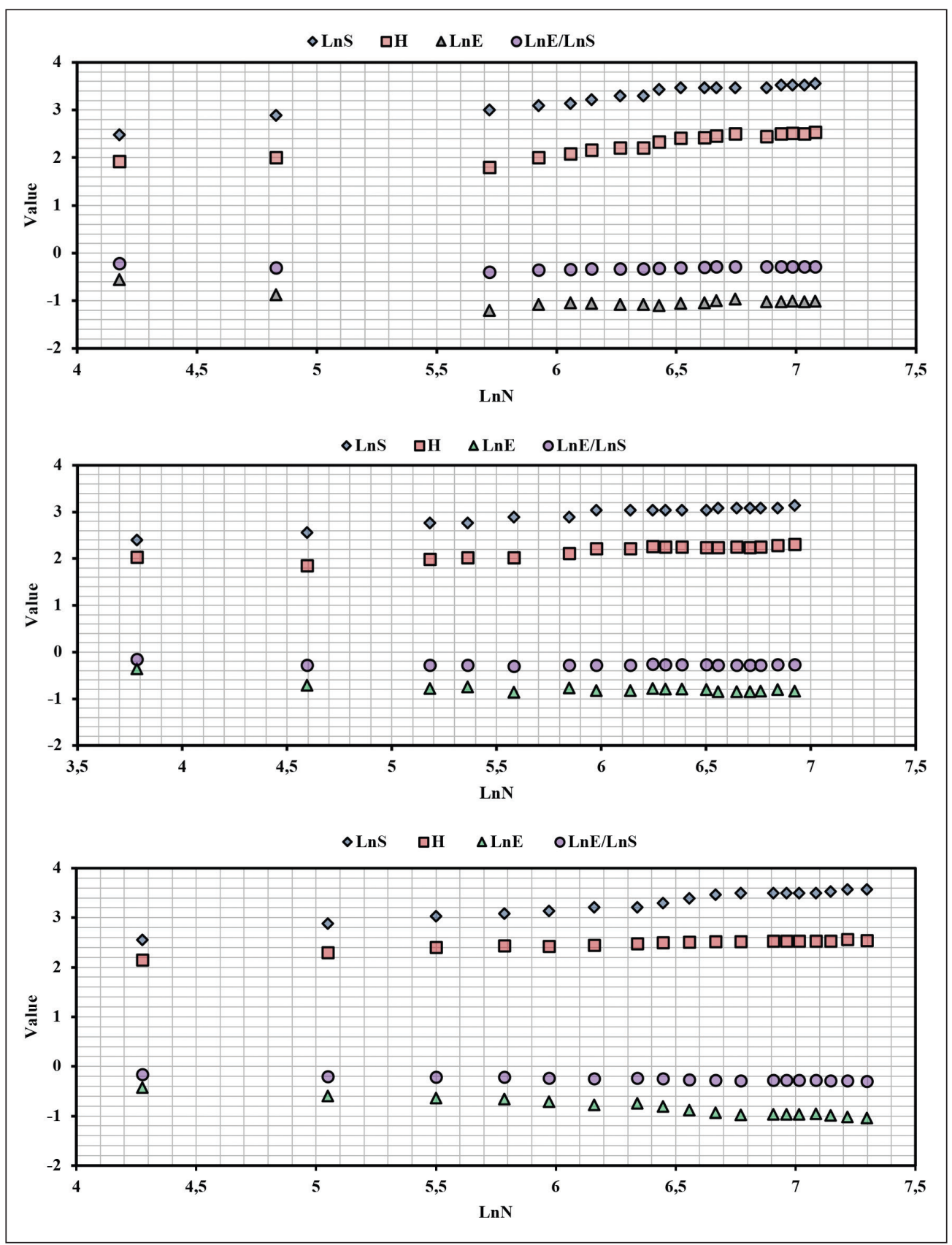

Figure 7. Plot of SHE analysis [S (species richness), $\mathrm{H}$ (information) and $\mathrm{E}$ (evenness) in the samples] calculated on relative abundance of 43 bird species of three different sites of (A) suburban; (B) urban; $(C)$ rural railway station areas. These represent the turnover of species between sites

7. ábra Az SHE elemzés eredményei ( $\mathrm{S}$ - fajgazdagság, $\mathrm{H}$ - információtartalom, $\mathrm{E}$ - egyenletesség) 43 madárfaj relatív abundancia adatainak felhasználásával. A - elővárosi, $B$ - városi, $C$ - vidéki területek 


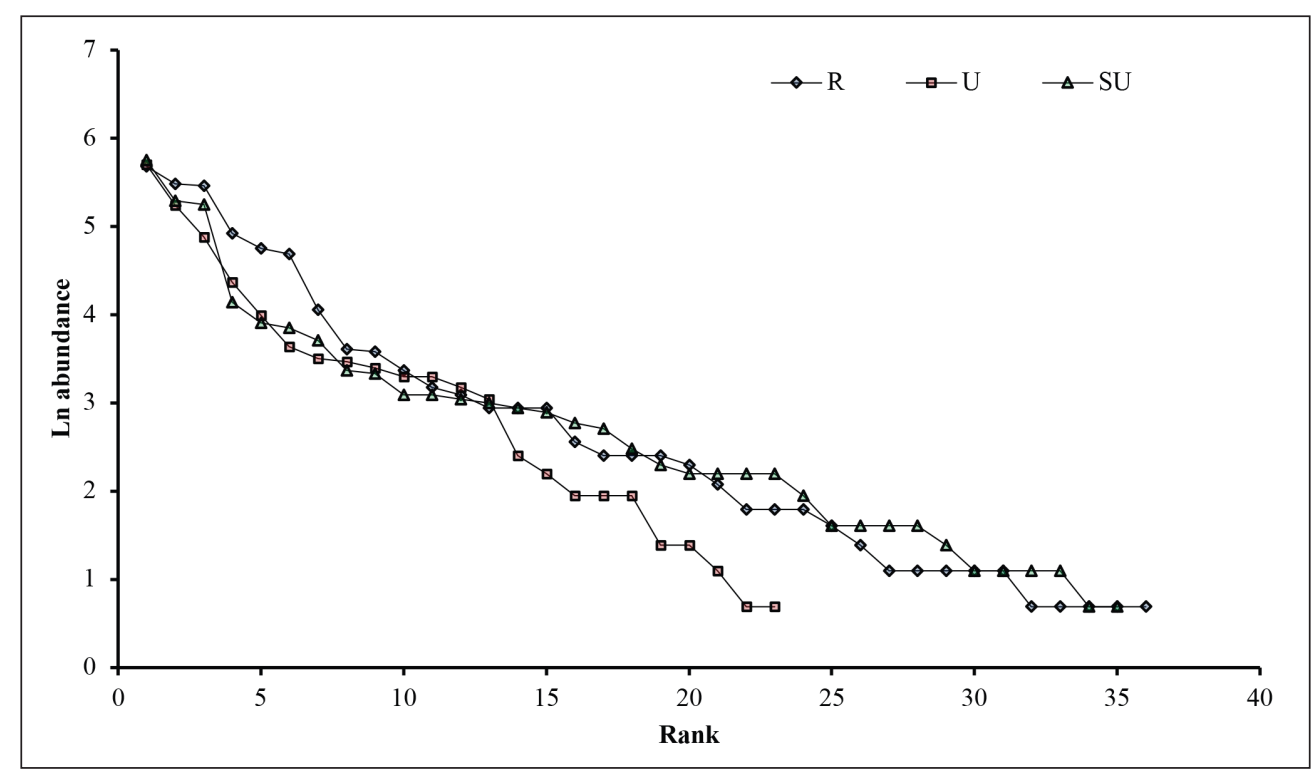

Figure 8. Rank-abundance curve of log $(n+1)$ transformed data of bird species abundance were used to show the rank of bird in suburban, urban and rural sites

8. ábra Rank-abundancia görbe az elővárosi (SU), vidéki (R) és városi (U) madárfajok összehasonlítására

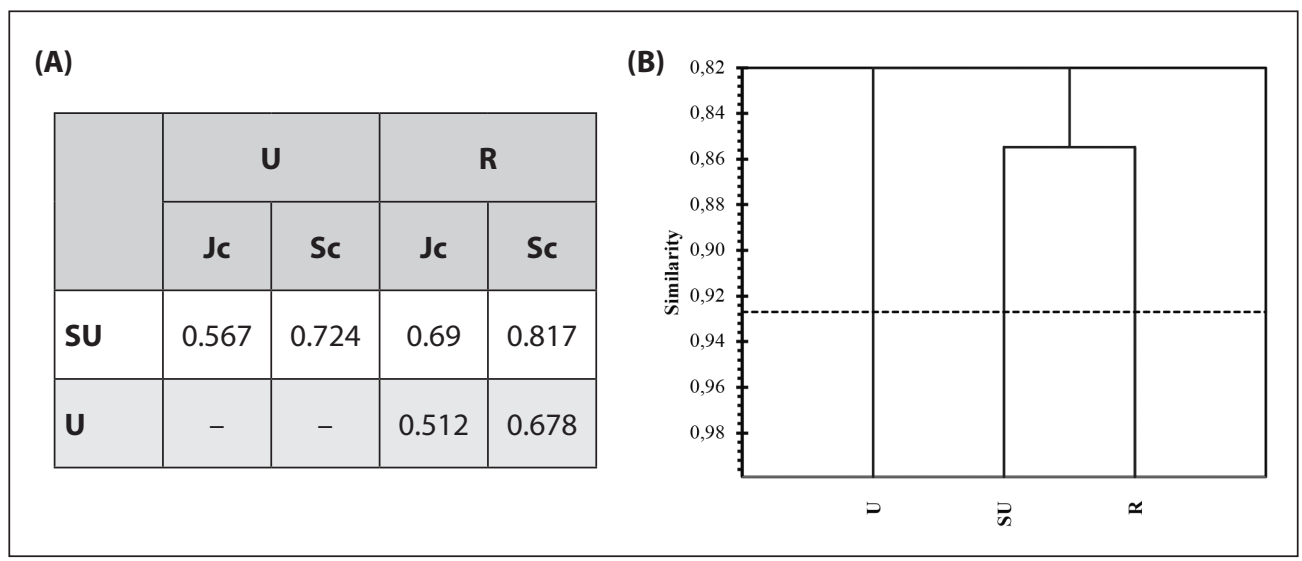

Figure 9. (A) Sorenson's (Sc) and Jaccard's (Jc) similarity coefficients calculated for different habitats in the study area $(\mathrm{U}=$ Urban station areas, $\mathrm{SU}=$ Suburban station areas and $\mathrm{R}=$ Rural station areas); (B) Hierarchical cluster analysis of similarity in species composition among the three study areas ( $\mathrm{SU}, \mathrm{U}$ and $\mathrm{R})$

9. ábra (A) Sorenson és Jaccard hasonlósági koefficiensek a három területtípusban. (B) A hierarchikus klaszterelemzés eredménye. U - városi terület, SU - elővárosi terület, $\mathrm{R}$ - vidéki terület 


\section{Feeding guilds of birds}

Analysis of feeding guilds revealed that among the 43 species observed during the entire study period in selected railway stations irrespective of the urban-rural gradient, 16 species $(37.2 \%)$ were omnivorous, 10 (23.25\%) were insectivorous, 8 (18.6\%) were carnivorous, $4(9.3 \%)$ were granivorous, $3(6.98 \%)$ were frugivorous, and the remaining 2 bird species $(4.65 \%)$ were nectarivorous. Omnivores were the most dominant species in suburban $(42.86 \%)$, urban $(39.13 \%)$, and rural $(36.11 \%)$ rail stations, followed by insectivores, with $26.08 \%, 25.71 \%$ and $22.22 \%$, for U, SU and R stations, respectively. Nonetheless, the carnivore bird species recorded in the rural, suburban and urban station areas were $19.44 \%, 14.28 \%$ and $13.04 \%$, respectively, while the granivorous species were highest in rural (17.4\%) regions as compared to urban $(8.57 \%)$ and suburban $(8.33 \%)$ regions. Nectarivorous species were recorded only from the suburban $(5.71 \%)$ and rural $(5.5 \%)$ railways (Figure 10).

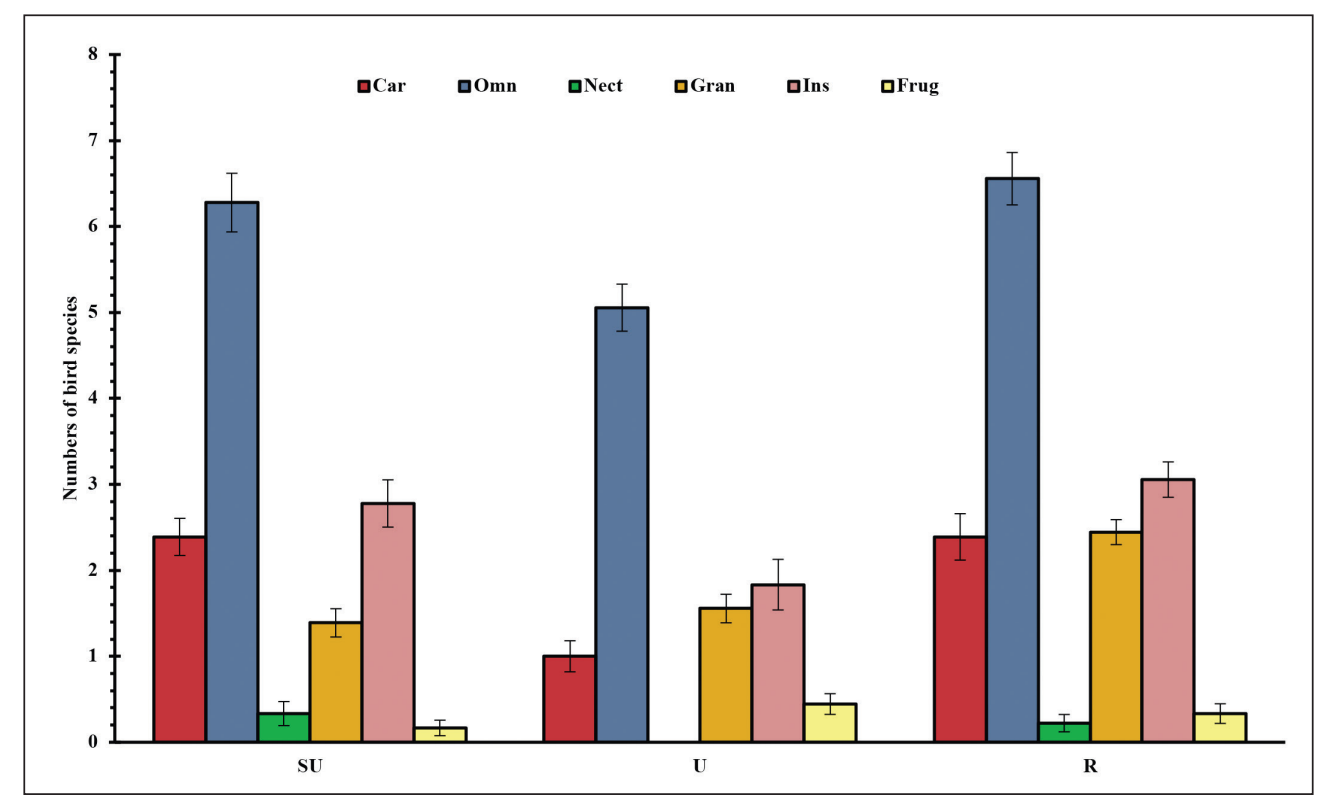

Figure 10. Numbers of bird species belong to six foraging guilds (i.e. Car=carnivore, Omn=omnivore, Nect=nectarivore, Gran=granivore, Ins=insectivore and Frug=Frugivore) recorded from the railway stations of suburban, urban and rural areas

10. ábra A hat különböző táplálkozási guildbe $(\mathrm{Car}=$ ragadozó, Omn $=$ mindenevő, Nest $=$ nektárfogyasztó, Gran = magevő, Ins = rovarevő, Frug = gyümölcsevő) tartozó madárfajok száma a három különböző területen vizsgált vasútállomásokon (SU - elővárosi, U - városi, $\mathrm{R}$ - vidéki) 


\section{Discussion}

The assessment of the bird diversity in the railway stations provided imperative information about the species assemblage patterns, variations in species composition, and species-specific abundance and richness in the station areas, much in support of the positive effects of railways on bird assemblages (Li et al. 2010, Morelli et al. 2014, Wiącek et al. 2015, 2019, 2020, Kaiser-Bonk et al. 2019). Variations in the bird species composition along the rural-urban gradient context remained similar to the observations made from Kolkata (Sengupta et al. 2014, Pal et al. 2019) and Amravati (Kale et al. 2018a, 2018b), India. During the entire study period, a total of 43 bird species belonging to 12 orders and 26 families were recorded in the railway stations irrespective of the urban-rural gradient, with the dominance of representatives from Passeriformes. The dominance of birds from Sturnidae, Corvidae, Columbidae, and Ardeidae families was observed in each area. While, the relative abundance of Sturnidae and Columbidae were higher in rural railway stations, the abundance of Corvidae was higher in suburban railway station areas. The House Crow (Corvus splendens) and the Common Myna (Acridotheres tristis) were the predominant species in all railway stations. Considering global population trend of birds, 4 species of urban areas, 6 species in suburban areas and 8 species from rural areas exhibited a decreasing trend in abundance. However, further monitoring is required to confirm the reasons for the decline in the abundance of these bird species. The species richness and the diversity indices of the bird assemblages were highest for the rural railway stations followed by the railway stations of suburban and urban areas. The Jaccard and Sorensen similarity coefficients revealed that the railway stations from the suburban and rural landscapes were the regions with the most similar species composition. In contrast, the rural and urban rail stations were the least similar in terms of species composition. Such patterns appear to be similar to the observations made from Kolkata (Sengupta et al. 2014, Pal et al. 2019) and Amravati (Kale et al. 2018a, 2018b), where the bird species richness were higher in rural areas contrast to the urban areas. In all these studies, a nestedness (Sengupta et al. 2014, Kale et al. 2018a, Pal et al. 2019) pattern were observed where the majority of the species of the urban areas were part of bigger species-rich assemblages of rural areas. Likewise, in the present instance, the species commonness between the rural and urban areas was observed, reflected through the indices of community similarity. Changes in the relative abundance of feeding guild of one bird may influence the abundance of others and thus, affects the community composition of the ecosystem (Barik et al. 2019). In this study, we found relatively higher abundance of omnivores in each selected railway stations irrespective of the urban-rural gradient followed by insectivores, while the abundance of other four guilds were the least. In the railway tracks near the forest areas, the insectivores dominate owing to the abundance of the macroinvertebrates like insects, more likely due to the edge effect (Wiącek et al. 2020). The granivorous bird species were the most abundant in rural railway stations as compared to urban and suburban station regions. A possible reason might be the presence of the agricultural landscapes along the railway tracks in the rural regions (Hossian \& Aditya 2016). The nectarivorous species were recorded only from the suburban and rural railway station areas and were completely missing in the urban railway stations. Positive effects of manmade infrastructures in course of urbanization on the reproductive success of some wildlife population were 
reported previously (Cardilini et al. 2013). The animals usually preferred to use the roadside constructions and associated structures have the physical and cognitive abilities to endure the possible risk (Fahrig \& Rytwinski 2009). Utilization of small territory size, ability to avoid conflict with human activities, high productiveness could provide those animals the adaptability to survive with the anthropogenic interferences. Reduction of predation pressure, occurrence of roadside agriculture practices (Sundar \& Kittur 2013, Hossain \& Aditya 2016) as well as abundance of vegetation (Morelli et al. 2014, Wiącek et al. 2015, 2019, 2020, Kaiser-Bonk et al. 2019) provide foraging and nesting habitats to make a unique ecological corridor. The power lines, over bridge pillars, station railing borders, lamp posts possibly provide the suitable sites for chasing the prey by carnivores and insectivores (Morelli et al. 2014). The anthropogenic constructions somehow attract a large number of opportunist bird species by offering environmental heterogeneity (Morelli et al. 2014, Kaiser-Bonk et al. 2019, Wiącek et al. 2019, 2020). In this study, it was evident that the anthropogenic structures can be utilized to attract species too effectively to increase diversity irrespective to urban-rural gradient. The vegetation near railway station areas acting as ecological corridors for many insects, made these areas suitable for foraging habitat for many insectivorous bird species. It was reported that the passerine species used artificial light along the railway station areas, which increase their activity period. The passerines also used warm surface of station grounds, which could conserve their metabolic energy. Besides, the shrubs, lamp posts, bridges in station areas provide better place to construct nests for these species (Morelli et al. 2014). Construction along the railway tract, mainly the electric wires and poles provide suitable place for displaying and singing for the passerines. For granivorous bird species the availability of small stones near railway track could be a source for gastroliths and surface sand on station ground could help the passerine species to accomplish their sand-bathing, helpful for cleaning their feathers (Morelli et al. 2014). It was observed that the railway station areas are most suitable territory for rodents as they found it better ground for availability of food and safest hiding place. The availability of rodents and their traffic mortality turns the railway station area as a better foraging ground for carnivorous birds. During the study, it was reported that near the railway tracks, the relative abundance of omnivorous bird species were maximum irrespective of urban-rural gradient. It may be due to the fondness of bird species always to share ecotone environment. In addition to the open agricultural landscape along the railway track, which could provide excellent foraging ground due to the better insulation, higher temperature in station areas reinforce the growth and availability of rodents and invertebrates (Delgado et al. 2007). The abundance of various kinds of food along the railway station areas due to the existence of special microclimatic conditions might be qualified as an anthropogenic construction suitable for various bird species occupying different feeding guilds along with the dominance of omnivores due to their inclination towards the marginal habitats, which could provide them an ecotone environment (Delgado et al. 2007, Barbaro et al. 2014). Diverse and more affluent vegetation near the railway tracks provide most favourable habitat for a large number of invertebrate populations (Vermeulen 1994), which attracted various insectivorous birds species towards that ecotone environment. Though the noise from busy roadside traffic provides negative impact on many bird species (Rheindt 2003, Summers et al. 2011), in our study, we did not observe any negative interactions with the train movements to their surroundings. Such bird species frequently 
observed in station areas did not react much in response to the noise made by the movement of trains through the station (Wiącek et al. 2015, 2019, 2020).

In the present observations, the railway stations across urban-rural gradient appear to bear a positive impact on the bird species assemblages. Considerable extent of taxonomic and functional diversity of birds was observed across the urban-rural gradient with prominent differences in the rural against the urban areas. Such observations tally with the characteristic bird species assemblages in urban areas (Pollack et al. 2017, Rodriguez et al. 2018, Filloy et al. 2019) and the differences in the urban and rural context (Sengupta et al. 2014, Kale et al. 2018a, 2018b, Pal et al. 2019). In the present study, higher diversity and species richness were observed in thesuburban and rural station areas than that of urban areas. Higher species richness and diversity index in periurban and suburban landscape as well as railway construction suggests human constructions are attractive to numerous bird species (Sandilyan \& Sudha 2013). On the basis of the habitat requirements, species can be divided into two groups. Some species that are highly accustomed to human activities and those with special habitat requirements (Fernández-Juricic 2000). Birds accustomed to stay with the human activities are not usually afraid of human activities rather they usually preferred such constructions like railway stations, bridges, light posts, which potentially have positive effects on their population to provide nesting sites, alternative feeding habitat increase habitat heterogeneity to support more species. Perhaps, these factors contributed to the abundance of the birds in the railway stations observed in the present instance, where, densely populated areas or agricultural landscapes in the adjacent regions were a redundant feature. As an extension to the present observation, the biotic homogenization (Pal et al. 2019) and the nestedness pattern (Sengupta et al. 2014) of the bird species assemblages in the railway associated landscapes can be explored to promote sustenance of the birds in the concerned spaces. Nonetheless, the present study substantiates the observations made on the railways associated bird species assemblages in different geographical locations (Morelli et al. 2014, Kaiser-Bonk et al. 2019, Wiącek et al. 2015, 2019, 2020), justifying that railway transportation infrastructures may play more positive role in organizing the bird species assemblages than their negative impacts. However, a gradient of the urban-rural context was also prominent in the assemblage structure and the guild features of the birds occurring in different railway stations considered in the study. Observations of the present study provide a foundation to consider the railway infrastructure of West Bengal and similar regions of India to be a prospective organizer of the bird species assemblages and thus, suitable for conservation initiatives.

\section{Acknowledgement}

The authors acknowledge the constructive comments of two anonymous reviewers in enhancement of the quality of the manuscript to its present form. The authors express thanks and gratitude to Dr. Tibor Csörgö, Editor-in-Chief, for his kind cooperation and helpful suggestions in formatting the manuscript to the present form. The authors acknowledge the respective Heads, Department of Zoology, University of Calcutta and The University of Burdwan for the facilities provided, including DST-FIST, and UGC-UPE II, Government of India. 


\section{References}

Addinsoft, S. A. R. L. 2010. XLSTAT software, version 9.0. - Addinsoft, Paris, France

Aich, A. \& Mukhopadhyay, S. 2008. Comparison of avifauna at the edges of contrasting forest 475 patches in Western Ghat Hills of India. - The Ring 30: 71-79. DOI: 10.2478/v10050-008-0001-6

Ali, S. \& Ripley, S. D. 1980. Handbook of the birds of India and Pakistan, together with those of Bangladesh, Nepal, Bhutan, and Sri Lanka. - Oxford University Press, Delhi, India

Ali, S. 1996. The book of Indian birds. - Oxford University Press, USA

Barbaro, L., Giffard, B., Charbonnier, Y., van Halder, I. \& Brockerhoff, E. G. 2014. Bird functional diversity enhances insectivory at forest edges: a transcontinental experiment. - Diversity and Distributions 20(2): 149-159. DOI: $10.1111 /$ ddi.12132

Barik, S., Mazumder, S. \& Saha, G. K. 2019. Composition and richness of avifauna in a suburban landscape of mid West Bengal, India. - In: Sanyal, A. K. (eds.) Wings of Life, Seminar \& Public Lecture Series, pp. 178-207. The Asiatic Society, Kolkata, India

Basavarajappa, S. 2006. Avifauna of agro-ecosystems of Maidan area of Karnataka. - Zoos' Print Journal 21(4): 2217-2219. DOI: 10.11609/JoTT.ZPJ.1277.2217-9

Beissinger, S. R. \& Osborne, D. R. 1982. Effects of urbanization on avian community organization. - The Condor 84(1): 75-83. DOI: 10.2307/1367825

Benítez-López, A., Alkemade, R. \& Verwij, P. A. 2010. The impacts of roads and other infrastructure on mammal and bird populations: A meta-analysis. - Biological Conservation 143(6): 1307-1316. DOI: 10.1016/j. biocon.2010.02.009

Bibby, C. J. 1999.Making the most of birds as environmental indicators. - Ostrich 70(1): 81-88. DOI: 10.1080/00306525.1999.9639752

Bibby, C. J., Burgess, N. D., Hill, D. A. \& Mustoe, S. 2000. Bird census techniques. $2^{\text {nd }}$ ed. - Elsevier, Academic Press, UK., pp. 302.

Blair, R. B. 1996. Land use and avian species diversity along an urban gradient. - Ecological Applications 6(2): 506-519. DOI: $10.2307 / 2269387$

Borad, C., Mukherjee, A. \& Parasharya, B. 2001. Damage potential of Indian SarusCrane in paddy crop agroecosystem in Kheda district Gujarat, India. - Agriculture, Ecosystems \& Environment 86(2): 211-215. DOI: 10.1016/S0167-8809(00)00275-9

Borda-de-Água, L., Barrientos, R., Beja, P. \& Pereira, H. M. 2017. Railway ecology. - In: Borda-de-Água, L., Barrientos, R., Beja, P. \& Pereira, H. M. (eds.) Railway Ecology. - Springer, Cham, pp. 3-7.

Bradford, D. F., Franson, S. E., Neale, A. C., Heggem, D. T., Miller, G. R. \& Canterbury, G. E. 1998. Bird species assemblages as indicators of biological integrity in Great Basin rangeland. - Environmental Monitoring and Assessment 49(1): 1-22. DOI: 10.1023/A:1005712405487

Browder, S. F., Johnson, D. H. \& Ball, I. 2002. Assemblages of breeding birds as indicators of grassland condition. - Ecological Indicators 2(3): 257-270. DOI: 10.1016/S1470-160X(02)00060-2

Buckland, S. T., Anderson, D. R., Burnham, K. P. \& Laake, J. L. 1993. Distance sampling: estimating abundance of biological populations. - Chapman and Hall, London

Buzas, M. A. \& Hayek, L. A. 1998. SHE analysis for biofacies identification. - The Journal of Foraminiferal Research 28(3): 233-239.

Canterbury, G. E., Martin, T. E., Petit, D. R., Petit, L. J. \& Bradford, D. F. 2000. Bird communities and habitat as ecological indicators of forest condition in regional monitoring. - Conservation Biology 14(2): 544558. DOI: $10.1046 / \mathrm{j} .1523-1739.2000 .98235 . x$

Cardilini, A. P., Weston, M. A., Nimmo, D. G., Dann, P. \& Sherman, C. D. H. 2013. Surviving in sprawling suburbs: suburban environments represent high quality breeding habitat for a widespread shorebird. - Landscape Urban Planning 115: 72-80. DOI: 10.1016/j.landurbplan.2013.04.001

Chace, J. F. \& Walsh, J. J. 2006.Urban effects on native avifauna: a review. - Landscape and Urban Planning 74(1): 46-69. DOI: 10.1016/j.landurbplan.2004.08.007

Chamberlain, D. E., Cannon, A. R., Toms, M., Leech, D. I., Hatchwell, B. \& Gaston, K. 2009. Avian productivity in urban landscapes: a review and meta-analysis. - Ibis 151(1): 1-18. DOI: 10.1111/j.1474919X.2008.00899.x

Chatterjee, A., Adhikari, S., Barik, A. \& Mukhopadhyay, S. K. 2014. The mid-winter assemblage and diversity of bird populations at Patlakhawa Protected Forest, Coochbehar, West Bengal, India. - The Ring 35(1): 31-53. DOI: 10.2478/ring-2013-0002 
DeGregorio, B. A., Weatherhead, P. J. \& Sperry, J. H. 2014. Power lines, roads, and avian nest survival: effects on predator identity and predation intensity. - Ecology and Evolution 49(9): 1589-1600. DOI: 10.1002/ece3.1049

del Hoyo, J., Elliott, A. \& Sargatal, J. 1996. Handbook of the Birds of the World, Vol. 3. Hoatzin to Auks. - Lynx Edicions, Barcelona

Delgado, J. D., Arroyo, N. L., Arevalo, J. R. \& Fernandez-Palacios, J. 2007. Edge effect of roads on temperature, light, canopy cover, and canopy height in laurel and pine forests. - Landscape and Urban Planning 81(4): 328340. DOI: 10.1016/j.landurbplan.2007.01.005

Dhindsa, M. S. \& Saini, H. K. 1994. Agricultural ornithology: an Indian perspective. - Journal of Biosciences 19(4): 391-402. DOI: 10.1007/BF02703176

Eeva, T., Tanhuanpää, S., Råbergh, C., Airaksinen, S., Nikinmaa, M. \& Lehikoinen, E. 2000. Biomarkers and fluctuating asymmetry as indicators of pollution-induced stress in two hole-nesting passerines. - Functional Ecology 14(2): 235-243. DOI: 10.1046/j.1365-2435.2000.00406.x

Fahrig, L. \& Rytwinski, T. 2009. Effects of roads on animal abundance: an empirical review and synthesis. - Ecology and Society 14(1): 1-20.

Fernández-Juricic, E. 2000. Avifaunal use of wooded streets in an urban landscape. - Conservation Biology 14(2): 513-521. DOI: 10.1046/j.1523-1739.2000.98600.x

Filloy, F., Zurita, G. A. \& Bellocq, M. I. 2019. Bird diversity in urban ecosystems: the role of the biome and land use along urbanization gradients. - Ecosystems 22(1): 213-227. DOI: 10.1007/s10021-018-0264-y

Fraixedas, S., Lindén, A., Piha, M., Cabeza, M. \& Gregory, R. 2020. A state-of-the-art review on birds as indicators of biodiversity: advances, challenges and future directions. - Ecological Indicators 118: 106728. DOI: 10.1016/j.ecolind.2020.106728

Gering, J. C. \& Blair, R. B. 1999.Predation on artificial bird nests along an urban gradient: predatory risk or relaxation in urban environments? - Ecography 22(5): 532-541. DOI: 10.1111/j.1600-0587.1999.tb01283.x

Gilbert, O. 2012. The Ecology of Urban Habitats. - Springer Science \& Business Media

Gregory, R. D., Noble, D., Field, R., Marchant, J., Raven, M. \& Gibbons, D. W. 2003.Using birds as indicators of biodiversity. - Ornis Hungarica 12-13: 11-24.

Grimmett, R., Inskipp, C. \& Inskipp, T. 2016. Birds of the Indian Subcontinent: India, Pakistan, Sri Lanka, Nepal, Bhutan, Bangladesh and the Maldives. - Bloomsbury India, pp. 448.

Haggard, W. H. 1990. Urban weather. - International Journal of Environmental Studies 36(1-2): 73-82. DOI: $10.1080 / 00207239008710584$

Haskell, D. G., Knupp, A. \& Schneider, M. 2001. Nest predator abundance and urbanization. - In: Marzluff, J. M., Bowman, R. \& Donnelly, R. (eds.) Avian Ecology and Conservation in an Urbanizing World. - Springer, New York, pp. 243-258.

Hossain, A. \& Aditya, G. 2016. Avian diversity in agricultural landscape: records from Burdwan, West Bengal, India. - Proceedings of the Zoological Society 69(1): 38-51. DOI: 10.1007/s12595-014-0118-3

Hutto, R. L., Pletschet, S. M. \& Hendricks, P. 1986. A fixed-radius point count method for nonbreeding and breeding season use. - The Auk 103(3): 593-602. DOI: 10.1093/auk/103.3.593

Issa, M. A. 2019. Diversity and abundance of wild birds species' in two different habitats at Sharkia Governorate, Egypt. - The Journal of Basic and Applied Zoology 80(1): 1-7. DOI: 10.1186/s41936-019-0103-5

Kaiser-Bonk, J., Skórka, P., Bonk, M., Lenda, M., Rożej-Pabijan, E., Wantuch, M. \& Moroń, D. 2019. The effect of railways on bird diversity in farmland. - Environmental Science and Pollution Research 26(30): 31086-31098. DOI: $10.1007 / \mathrm{s} 11356-019-06245-0$

Kale, M., Ferrante, M., Dudhe, N., Kasambe, R., Trukhanova, I. S., Ivanova, T., Bhattacharya, P. \& Lövei, G. I. 2018a Nestedness of bird assemblages along an urbanisation gradient in Central India. - Journal of Urban Ecology 4(1): 1-8. DOI: 10.1093/jue/juy017

Kale, M., Dudhe, N., Ferrante, M., Ivanova, T., Kasambe, R., Trukhanova, I. S., Bhattacharya, P. \& Lövei, G. I. $2018 \mathrm{~b}$ The effect of urbanization on the functional and scale-sensitive diversity of bird assemblages in Central India. - Journal of Tropical Ecology 34(6): 341-350. DOI: 10.1017/S0266467418000317

Khan, S. \& Naher, H. 2009. Birds in Kurigram district of Bangladesh. - Journal of Threatened Taxa 1(4): 245-250. DOI: $10.11609 /$ JoTT.o1698.245-50

Koskimies, P. 1989. Birds as a tool in environmental monitoring. - Annales Zoologici Fennici 26: $153-166$.

Krebs, C. J. 1999. Ecological methodology, $2^{\text {nd }}$ ed. - Addison Wesley Longman, Menlo Park, CA.

Kumar, A., Tak, P. C. \& Sati, J. P. 2006. Residential, population and conservation status of Indian wetland birds. In: Boere, G. C., Galbraith, C. A. \& Stroud, D. A. (eds.) Waterbirds Around the World. - The Stationery Office, Edinburgh, UK., pp. 308-309. 
Kumar, P. \& Gupta, S. 2013. Status of wetland birds of Chhilchhila Wildlife Sanctuary, Haryana, India. - Journal of Threatened Taxa 5(5): 3969-3976. DOI: 10.11609/JoTT.o3158.3969-76

Lee, P. F., Ding, T. S., Hsu, F. H. \& Geng, S. 2004. Breeding bird species richness in Taiwan: distribution on gradients of elevation, primary productivity and urbanization. - Journal of Biogeography 31(2): 307-314. DOI: 10.1046/j.0305-0270.2003.00988.x

Li, Z., Ge, C., Li, J., Li, Y., Xu, A., Zhou, K. \& Xue, D. 2010. Ground-dwelling birds near the Qinghai-Tibet highway and railway. - Transportation Research Part D: Transport and Environment 15(8): 525-528. DOI: 10.1016/j.trd.2010.07.004

Ludwig, J. A., Quartet, L., Reynolds, J. F. \& Reynolds, J. F. 1988. Statistical ecology: a primer in methods and computing, Vol. 1. - Wiley-Interscience Pub., New York

Magurran, A. E. 1988. Ecological diversity and its measurement. - Chapman and Hall, London

Margalef, R. 1958. Information theory in biology. - General Systems Yearbook 3: 36-71.

Marzluff, J. M. 2001. Worldwide urbanization and its effects on birds. - In: Marzluff, J. M., Bowman, R. \& Donnelly, R. (eds.) Avian Ecology and Conservation in an Urbanizing World. - Springer, Boston, MA, USA. DOI: $10.1007 / 978-1-4615-1531-9 \_2$

McAleece, N., Gage, J. D. \& Lambshead, J. 1997. Biodiversity Professional. - The Natural History Museum \& The Scottish Association for Marine Science, UK.

Melles, S., Glenn, S. \& Martin, K. 2003. Urban bird diversity and landscape complexity: species-environment associations along a multiscale habitat gradient. - Conservation Ecology 7(1): 5. DOI: 10.5751/ES-00478070105

Morelli, F., Beim, M., Jerzak, L., Jones, D. \& Tryjanowski, P. 2014. Can roads, railways and related structures have positive effects on birds? - A review. - Transportation Research Part D: Transport and Environment 30: 21-31. DOI: 10.1016/j.trd.2014.05.006

Mukherjee, S., Banerjee, S., Saha, G. K., Basu, P. \& Aditya, G. 2015. Butterfly diversity in Kolkata, India: An appraisal for conservation management. - Journal of Asia-Pacific Biodiversity 8(3): 210-221. DOI: 10.1016/j. japb.2015.08.001

Pain, D. J., Gargi, R., Cunningham, A. A., Jones, A. \& Prakash, V. 2004.Mortality of globally threatened Sarus Cranes Grus antigon from monocrotophos poisoning in India. - Science of the Total Environment 326(1-3): 55-61. DOI: 10.1016/j.scitotenv.2003.12.004

Pal, M., Pop, P., Mahapatra, A., Bhagat, R. \& Hore, U. 2019. Diversity and structure of bird assemblages along urban-rural gradient in Kolkata, India. - Urban Forestry \& Urban Greening 38(1): 84-96. DOI: 10.1016/j. ufug.2018.11.005

Pereira, H. M., Navarro, L. M. \& Martins, I. S. 2012. Global biodiversity change: the bad, the good, and the unknown. - Annual Review of Environment and Resources 37: 25-50. DOI: 10.1146/annurev-environ-042911-093511

Pollack, L., Ondrasek, N. R. \& Calisi, R. 2017. Urban health and ecology: the promise of an avian biomonitoring tool. - Current Zoology 63(2): 205-212. DOI: 10.1093/cz/zox011

Praveen, J., Jayapal, R. \& Pittie, A. 2016. A checklist of the birds of India. - Indian Birds 11(5-6): 113-172.

Profillidis, V. A. 2006. Railway Management and Engineering. - Ashgate Publishing Company

Rheindt, F. E. 2003. The impact of roads on birds: does song frequency play a role in determining susceptibility to noise pollution? - Journal für Ornithologie 144(3): 295-306. DOI: 10.1007/BF02465629

Rodrigues, A. G., Borges-Martins, M. \& Zilio, F. 2018. Bird diversity in an urban ecosystem: the role of local habitats in understanding the effects of urbanization. - Iheringia Série Zoologia 108(11): e2018017. DOI: $10.1590 / 1678-4766 \mathrm{e} 2018017$

Sandilyan, S. \& Sudha, M. 2013. Check list of avifauna in between Kutralam and Narasingampattai Railway Track, Tamilnadu, India. - International Journal of Pure and Applied Zoology 1(1): 7-14.

Şekercioğlu, C. H. 2012a Bird functional diversity and ecosystem services in tropical forests, agroforests and agricultural areas. - Journal of Ornithology 153(1): 153-161. DOI: 10.1007/s10336-012-0869-4

Şekercioğlu, C. H. 2012b Promoting community-based bird monitoring in the tropics: Conservation, research, environmental education, capacity-building, and local incomes. - Biological Conservation 151(1): 69-73. DOI: 10.1016/j.biocon.2011.10.024

Sengupta, S., Mondal, M. \& Basu, P. 2014. Bird species assemblages across a rural urban gradient around Kolkata, India. - Urban Ecosystems 17(2): 585-596.

Sorace, A. 2002. High density of bird and pest species in urban habitats and the role of predator abundance. - Ornis Fennica 79(2): 60-71. 
Summers, P. D., Cunnington, G. M. \& Fahrig, L. 2011. Are the negative effects of roads on breeding birds caused by traffic noise? - Journal of Applied Ecology 48(6): 1527-1534. DOI: 10.1111/j.1365-2664.2011.02041.x

Sundar, K. G. \& Kittur, S. 2013. Can wetlands maintained for human use also help conserve biodiversity? Landscape-scale patterns of bird use of wetlands in an agricultural landscape in north India. - Biological Conservation 168: 49-56. DOI: 10.1016/j.biocon.2013.09.016

Vallecilo, S., Maes, J., Polce, C. \& Lavalle, C. 2016. A habitat quality indicator for common birds in Europe based on species distribution models. - Ecological Indicators 69: 488-499. DOI: 10.1016/j.ecolind.2016.05.008

van der Ree, R., Jaeger, J. A., van der Grift, E. A. \& Clevenger, A. P. 2011. Effects of roads and traffic on wildlife populations and landscape function: road ecology is moving toward larger scales. - Ecology and Society 16(1): 48. URL: http://www.ecologyandsociety.org/vol16/iss1/art48/

Venier, L. A. \& Pearce, J. L. 2004.Birds as indicators of sustainable forest management. - The Forestry Chronicle 80(1): 61-66. DOI: $10.5558 / \mathrm{tfc} 80061-1$

Veríssimo, D., Fraser, I., Groombridge, J., Bristol, R. \& MacMillan, D. C. 2009. Birds as tourism flagship species: a case study of tropical islands. - Animal Conservation 12(6): 549-558. DOI: $10.1111 /$ j.14691795.2009.00282.x

Vermeulen, H. J. 1994. Corridor function of a road verge for dispersal of stenotopic heathland ground beetles Carabidae. - Biological Conservation 69(3): 339-349. DOI: 10.1016/0006-3207(94)90433-2

Whelan, C. J., Wenny, D. G. \& Marquis, R. J. 2008. Ecosystem services provided by birds. - Annals of the New York Academy of Sciences 1134(1): 25-60. DOI: 10.1196/annals.1439.003

Wiącek, J., Polak, M., Filipiuk, M., Kucharczyk, M. \& Bohatkiewicz, J. 2015. Do birds avoid railroads as has been found for roads? - Environmental Management 56(3): 643-652. DOI: 10.1007/s00267-015-0528-7

Wiącek, J., Polak, M., Filipiuk, M. \& Kucharczyk, M. 2019. Does railway noise affect forest birds during the winter? - European Journal of Forest Research 138(5): 907-915. DOI: 10.1007/s10342-019-01212-3

Wiącek, J., Polak, M., Filipiuk, M., Kucharczyk, M. \& Dawidowicz, Ł. 2020. Do railway lines affect the distribution of woodland birds during autumn? - PLoS ONE 15(4): e0231301. DOI: 10.1371/journal.pone.0231301

Williams, P. H., Burgess, N. D. \& Rahbek, C. 2000. Flagship species, ecological complementarity and conserving the diversity of mammals and birds in sub-Saharan Africa. - Animal Conservation 3(3): 249-260. DOI: 10.1111/j.1469-1795.2000.tb00110.x

Zar, J. H. 1999. Biostatistical analysis, $4^{\text {th }}$ ed. - Pearson Education Singapore Pte. Ltd., Indian Branch, New Delhi, India

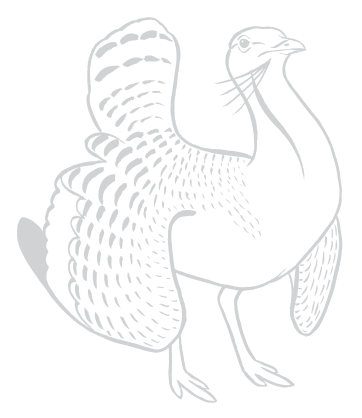

